\title{
1 Chromatin features define adaptive genomic regions in a 2 fungal plant pathogen
}

4 David E. Cook ${ }^{1,2^{*}}$, Martin Kramer ${ }^{2}$, Michael F. Seidl2,3, Bart PHJ Thomma ${ }^{2,4 *}$

$6{ }^{1}$ Department of Plant Pathology, Kansas State University, Manhattan KS, USA

7 2Laboratory of Phytopathology, Wageningen University \& Research, Wageningen,

8 The Netherlands

9 3Theoretical Biology \& Bioinformatics Group, Department of Biology, Utrecht

10 University, Utrecht, The Netherlands

$11{ }^{4}$ Cluster of Excellence on Plant Sciences (CEPLAS), University of Cologne, Botanical

12 Institute, Cologne, Germany

$13{ }^{*}$ Corresponding authors: David E. Cook, decook@ksu.edu; Bart PHJ Thomma,

\section{4 bart.thomma@wur.nl}




\section{ABSTRACT}

17 Understanding the complex information stored in a genome remains challenging

18 since multiple connected regulatory mechanisms act at various scales to determine

19 function. Increased comprehension of genome function at scales beyond contiguous

20 nucleotides will help understand genetic diseases, the emergence of pathogenesis,

21 and more broadly the genomics of adaptation. Here we report the analysis of DNA

22 methylation, histone modification, and DNA accessibility in the plant pathogenic

23 vascular wilt fungus Verticillium dahliae. Functional analysis details that DNA

24 methylation is restricted to repetitive elements, such as transposable element DNA,

25 but interestingly only some repetitive DNA is methylated. This incomplete DNA

26 methylation is associated with repetitive DNA residing in specific compartments of

27 the genome that were previously defined as Lineage-Specific (LS) regions. These

28 regions are hypervariable between $V$. dahliae isolates and contain genes that

29 support host colonization and adaptive traits. LS regions are associated with H3 Lys-

3027 methylated histones (H3K27me3), and repetitive DNA within LS regions are

31 more transcriptionally active and have increased DNA accessibility, representing a

32 hybrid chromatin state when compared to repetitive regions within the core

33 genome. We used machine learning algorithms trained on epigenetic and DNA

34 accessibility data to predict LS regions with high recall, identifying approximately

35 twice as much LS DNA in the $V$. dahliae genome as previously recognized.

36 Collectively, these results characterize LS regions in an intermediate chromatin

37 state and provide evidence that links chromatin features and genome architecture

38 to adaptive regions within the genome. 


\section{INTRODUCTION}

40 Genomes are not randomly organized and comprise complex information beyond

41 their linear nucleic acid sequence ${ }^{1}$. While scientific understanding of genome

42 biology continues to grow, significant efforts in the past decade have focused on

43 sequencing new species and additional genotypes of those species ${ }^{2}$. However, there

44 is a great need to decode the complex information stored in these genomes, to

45 understand genomic responses over various time scales, and ultimately to more

46 fully understand how genotypes lead to phenotypes. With the growing number of

47 high-quality, highly contiguous genome assemblies it is possible to analyze genome

48 organization into chromosomes at high resolution ${ }^{3}$. Present day genome

49 organization reflects evolutionary solutions to the challenges of information

50 processing and adaptation; a genome must faithfully pass vast amounts of

51 information across cell-cycles and reproduction, packaged into limited physical

52 space, while achieving correct access to the information in response to

53 developmental, environmental or chemical signals. In addition, there needs to be

54 appreciable stochastic genetic variation to ensure that phenotypic variation is

55 present for unknown future events. Organisms undergoing mainly asexual

56 reproduction face an additional evolutionary constraint as they must generate this

57 genetic variation in the absence of meiotic recombination ${ }^{4}$. Many economically

58 important fungal plant pathogens are either asexual or undergo more frequent

59 asexual reproduction compared to sexual reproduction ${ }^{5}$. Interestingly, fungal

60 pathogens are subject to additional evolutionary pressure from their hosts, as host-

61 pathogen interactions create dynamical systems with shifting, yet near-constant

62 selective pressure on the two genomes ${ }^{6}$. These attributes make plant-fungal

63 interactions a particularly interesting system to study aspects of genome evolution

64 and genome organization ${ }^{7,8}$.

65

66 Plant invading microbes use effectors to suppress, avoid or mitigate the plant

67 immune system ${ }^{9,10}$. Plants in-turn use a variety of plasma-membrane bound and

68 cytoplasmic receptors to recognize invasion, through recognition of the effector or 
69 its biochemical activity, creating a strong selective pressure on the microbe to

70 modify the effector or its function to alleviate recognition ${ }^{11,12}$. The plant pathogenic

71 fungus Verticillium dahliae causes vascular wilt diseases on hundreds of plant hosts.

72 V. dahliae is presumed asexual and generates genomic diversity in the absence of

73 sexual recombination through large-scale chromosome re-arrangements and

74 segmental duplications ${ }^{13-16}$. The regions undergoing such duplications and re-

75 arrangements are hypervariable between $V$. dahliae isolates, and consequently have

76 been referred to as Lineage-Specific (LS) regions. These LS regions are enriched for

77 in planta expressed genes and harbor many effector genes contributing to host

78 infection 14,17,18. Similar non-random genomic arrangement of effectors have been

79 reported across diverse plant pathogenic fungal and oomycete genomes ${ }^{14,19-25}$. One

80 summary of these observations is referred to as the two-speed genome, in which

81 repeat-rich regions harboring effectors evolve more rapidly than genes outside

82 these regions 26.

83

84 Previous research in various plant-associated fungi has established a link between

85 posttranslational histone modifications and transcriptional regulation of adaptive

86 trait genes. These genes include effectors that facilitate host infection, and

87 secondary metabolite (SM) clusters that code for genes that produce chemicals

88 important for niche fitness ${ }^{27}$. By removing or reducing enzymes responsible for

89 particular repressive histone modifications, such as di- and trimethylation of Lys9

90 and Lys27 residues of histone H3 (H3K9me2/3 and H3K27me2/3), a

91 disproportionally high number of effector and SM cluster genes are derepressed,

92 although a direct role of these marks in transcriptional control was not

93 demonstrated ${ }^{28-30}$. However, evidence from the fungus Epichloe festucae that forms

94 a mutualistic interaction with its grass host Lolium perenne indicates that direct

95 transcriptional regulation through histone modification dynamics is possible ${ }^{31}$.

96 Although there are clear indications that the epigenome (i.e. heritable chemical

97 modifications to DNA and histones not affecting the genetic sequence) plays a role in 
98 adaptive gene regulation, additional evidence is needed to fully understand this

99 phenomenon.

100

101 Epigenetic modifications influence chromatin structure, defined as the DNA-RNA-

102 protein interactions giving DNA physical structure in the nucleus ${ }^{32,33}$. This physical

103 structure affects how DNA is organized in the nucleus and DNA accessibility.

104 Methylation of H3K9 and H3K27 are hallmarks of heterochromatin; DNA that is

105 tightly compacted in the nucleus ${ }^{34-37}$. H3K9 methylation is not only associated with

106 controlling constitutive heterochromatin, but also tightly linked with DNA cytosine

107 methylation ( $\mathrm{mC}$ ), which serves as an epigenetic mark contributing to

108 transcriptional silencing ${ }^{38}$. A single DNA methyltransferase gene, termed Dim2,

109 performs cytosine DNA methylation in the saprophytic fungus Neurospora crassa ${ }^{39}$.

110 Histone methylation at H3K9 directs DNA methylation by DIM2 through another

111 protein, termed heterochromatin protein 1 (HP1), which physically associates with

112 both DIM2 and H3K9me3 ${ }^{40,41}$. Some fungi possess a unique pathway to limit the

113 expansion of repetitive DNA such as transposable elements through repeat-induced

114 point mutation (RIP), a mechanism that specifically mutates repetitive DNA in the

115 genome during meiosis and induces heterochromatin formation ${ }^{42,43}$. The mutations

116 occur at methylated cytosines resulting in conversion to thymines ( $\mathrm{C}$ to $\mathrm{T}$ mutation)

11744 . H3K27 methylation is associated with heterochromatin that is thought to be

118 more flexible in its chromatin status and exist as bivalent chromatin that may be

119 either transcriptionally repressed or active depending on developmental stage or

120 environmental cues ${ }^{45-48}$. The deposition of H3K27me3 is controlled by a histone

121 methyltransferase that is a member of a complex of proteins termed Polycomb

122 Repressive Complex 2 (PRC2), with orthologs of the core machinery present across

123 many eukaryotes ${ }^{36,49}$.

125 In addition to heterochromatin playing a role in transcriptional regulation in

126 filamentous fungi, epigenetic marks contributing to chromatin may influence

127 genome evolution ${ }^{50}$. In N. crassa, DNA is physically arranged in the nucleus 
128 corresponding to heterochromatic and euchromatic domains, with strong inter- and

129 intra-heterochromatin DNA-DNA interactions reported ${ }^{51,52}$. Recent experimental

130 evidence using Zymoseptoria tritici, a fungal pathogen of wheat, suggests that

131 H3K27me3 promotes genomic instability ${ }^{53}$. In the oomycete plant pathogens

132 Phytophthora infestans and Phytophthora sojae a clear association exists between

133 gene-sparse and transposon-rich regions of the genome and the occurrence of

134 adenine N6-methylation $(6 \mathrm{~mA}){ }^{54}$. Collectively these examples point towards an

135 unexplained connection between the epigenome, genome architecture, and adaptive

136 evolution. To examine the hypothesis that epigenetic modifications influence the

137 adaptive LS regions of $V$. dahliae, we performed a series of genetic, genomic, and

138 machine learning analyses to characterize these regions in greater detail.

\section{RESULTS}

\section{DNA cytosine methylation occurs at transposable elements}

142 To understand the role of DNA methylation in $V$. dahliae, whole-genome bisulfite

143 sequencing, in which unmethylated cytosine bases are converted to uracil while

144 methylated cytosines remain unchanged ${ }^{55,56}$, was performed in the wild-type and a

145 heterochromatin protein 1 deletion mutant ( $\Delta h p 1)$. The overall level of DNA

146 methylation in $V$. dahliae is low, with an average weighted methylation percentage

147 (calculated as the number of reads supporting methylation over the number of

148 cytosines sequenced) at CG dinucleotides of $0.4 \%$ (Table 1). The fractional CG

149 methylation level (calculated as the number of cytosine positions with a methylated

150 read over all cytosine positions) is higher, averaged to $9.7 \%$ over $10 \mathrm{~kb}$ windows.

151 Weighted and fractional cytosine methylation $(\mathrm{mC})$ levels are statistically

152 significantly higher in the WT compared to the $\Delta h p 1$ mutant for all cytosine contexts

153 (Table 1, Supplemental Fig. S1A and B). This result is consistent with the

154 requirement of HP1 for DNA methylation in $N$. crassa $^{40}$. To understand DNA

155 methylation in the context of the functional genome, DNA methylation was analyzed

156 over genes, promoters, and transposable elements (TE). Despite statistically

157 significant differences between WT and $\Delta h p 1$ for gene and promoter methylation, 
158 the bisulfite sequencing data shows virtually no DNA methylation at these two

159 features (Fig. 1A). We attribute the difference to a marginal set of elements having a

160 real difference between the genotypes, but the biological significance is likely

161 negligible (Fig. 1A). In contrast, there is a much higher degree of methylation, and a

162 notable difference between wild-type and $\triangle h p 1$ methylation levels at TEs (Fig. 1A,

163 bottom panel), with the average CG methylation level being five times higher in the

164 wild-type strain.

165

166

Table 1. Summary of DNA methylation in Verticillium dahliae wild-type (WT) and heterochromatin protein 1 deletion mutant $(\Delta h p 1)$ as measured by whole genome bisulfite sequencing calculated over $10 \mathrm{~kb}$ non-overlapping windows.

\begin{tabular}{ccccccc}
\hline Genotype & $\begin{array}{c}\text { Avg. } \\
\text { Weighted } \\
\text { mCG }\end{array}$ & $\begin{array}{c}\text { Avg. } \\
\text { Weighted } \\
\text { mCHG }\end{array}$ & $\begin{array}{c}\text { Avg. } \\
\text { Weighted } \\
\text { mCHH }\end{array}$ & $\begin{array}{c}\text { Avg. } \\
\text { Fraction } \\
\text { mCG }\end{array}$ & $\begin{array}{c}\text { Avg. } \\
\text { Fraction } \\
\text { mCHG }\end{array}$ & $\begin{array}{c}\text { Avg. } \\
\text { Fraction } \\
\text { mCHH }\end{array}$ \\
\hline WT & 0.0040 & 0.0037 & 0.0034 & 0.097 & 0.097 & 0.088 \\
$\Delta h p 1$ & 0.0030 & 0.0030 & 0.0032 & 0.082 & 0.083 & 0.079 \\
\hline
\end{tabular}

Avg. Weighted, The average of total methylated cytosines in a given context divided a specific context in a $10 \mathrm{~kb}$ window; $\mathrm{mCG}$, methylated cytosine residing next to a guanine; $\mathrm{mCHG}$, methylated cytosine residing next to any base that is not a guanine next to a guanine; $\mathrm{mCHH}$, methylated cytosine residing next to any two bases that are not a guanines.

177 To further analyze DNA methylation levels and confirm that the low DNA

178 methylation levels in the wild-type strain are indeed different than those in $\Delta h p 1, \mathrm{CG}$

179 DNA methylation levels were plotted in $10 \mathrm{~kb}$ windows across individual

180 chromosomes. These plots clearly show that DNA methylation is not continuously

181 present across the $V$. dahliae genome, and DNA methylation is significantly different

182 between wild-type and $\Delta h p 1$ (Fig. 1B, C). Furthermore, regions in the genome with

183 higher densities of TEs and lower gene numbers have higher levels of DNA

184 methylation, consistent with the global DNA methylation summary (Fig. 1B and C).

185 Interestingly, these results show that while DNA methylation is only present at TEs,

186 not all TEs are methylated, a phenomenon that was previously described as 'non-

187 exhaustive' DNA methylation ${ }^{57}$. To further understand this phenomenon, we sought 
188 to identify discriminating genomic features that could account for some TEs not

189 being methylated. The whole-chromosome methylation data suggested a lack of

190 DNA methylation at previously identified LS regions (Fig. 1C, grey windows). These

191 LS regions were previously detailed for $V$. dahliae, and are characterized as regions

192 that are highly variable between isolates of the species, are enriched for actively

193 transcribed TEs, and contain an increased proportion of genes involved in host

194 virulence ${ }^{13-15}$. Thus, we tested if DNA sequences at LS regions are less frequently

195 methylated by comparing weighted mCG levels in $10 \mathrm{~kb}$ bins containing at least one

196 TE for core versus LS regions. This analysis showed significantly more DNA

197 methylation for core bins, which cannot be accounted for by a simple difference in

198 the number of TEs in the core and LS regions analyzed (Fig. 1D and E). Higher CG

199 methylation levels also hold true when analyzed at the level of individual TE

200 elements (Fig. 1F, numbers of elements in Supplemental Table S1). Collectively,

201 these analyses demonstrate that DNA methylation occurs almost exclusively at TEs

202 and, importantly, that not all TEs are methylated. This observation can in part be

203 explained by mCG differences for TEs in the core versus LS regions.

A Gene Methylation
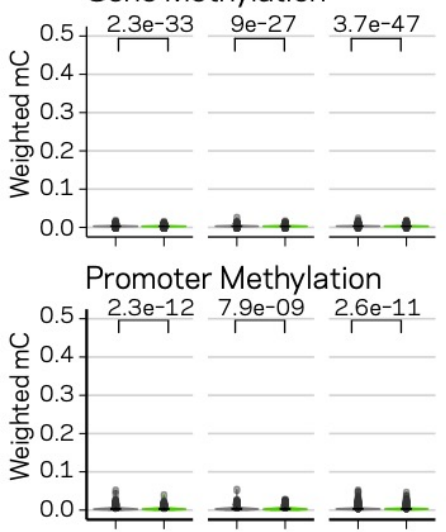

TE Methylation

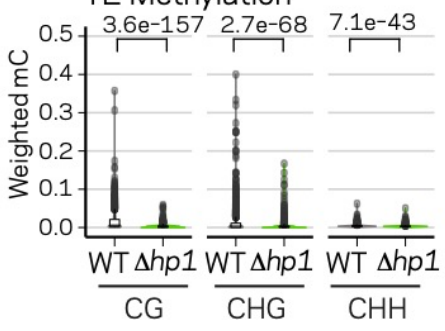

B
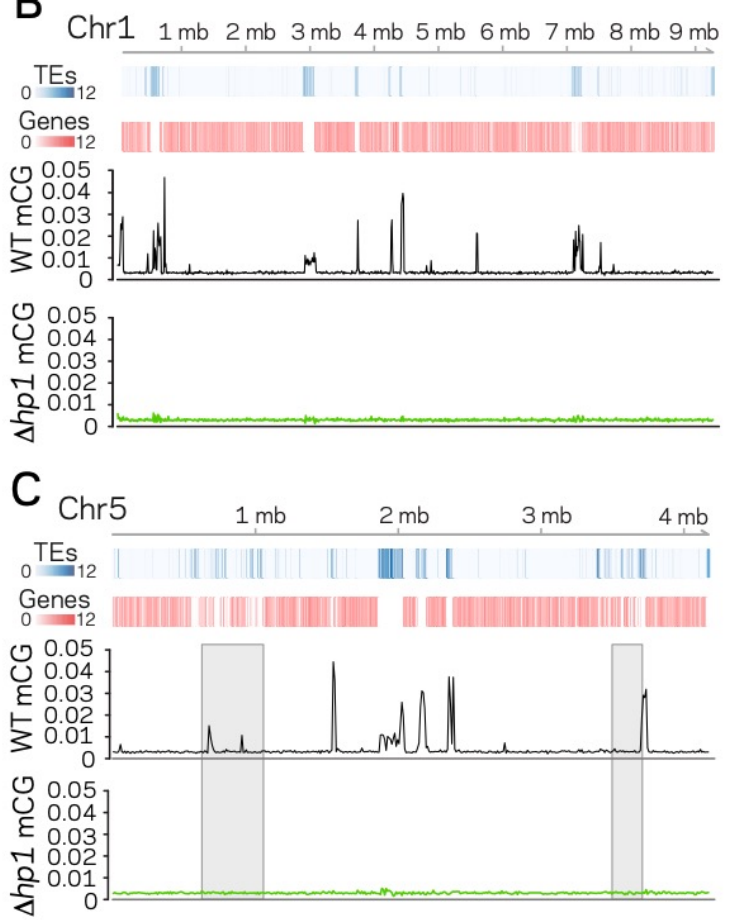

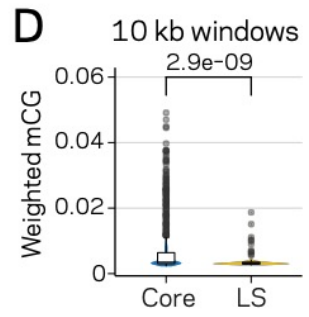

E $\quad 10 \mathrm{~kb}$ windows

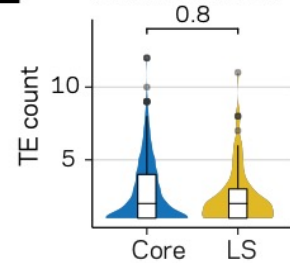

F Individual TEs

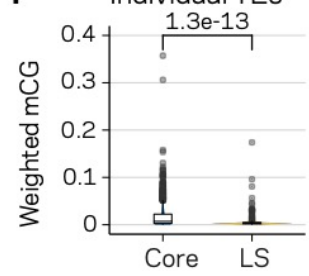


Figure 1. DNA methylation is only present at transposable elements, but not at those present in LS regions. (A) Violin plot of the distribution of DNA methylation levels quantified as weighted methylation over Genes, Promoters and TEs. Cytosine methylation was analyzed in the CG, CHG and CHH sequence context. Methylation was measured in the wild-type (WT) and heterochromatin protein 1 knockout strain $(\Delta h p 1)$. (B, C) Whole chromosome plots showing $\mathrm{TE}$ and Gene counts (blue and red heatmaps) and wild-type (black lines) and $\Delta h p 1$ (green line) CG methylation as measured with bisulfite sequencing. Data is computed in 10 kilobase non-overlapping windows. (C) Two previously defined LS regions (Faino et al. 2016) are highlighted by grey windows. (D) Violin plot of weighted cytosine methylation in $10 \mathrm{~kb}$ windows broken into core versus LS location (E) Same as D but plots are for the counts of TEs per $10 \mathrm{~kb}$ window. (F) Same as in D but methylation levels were computed at individual TE elements. Statistical differences for indicated comparisons were carried out using nonparametric Mann-Whitney test with associated p-values shown.

206 Transposable element classes have distinct profiles for genomic and

\section{7 epigenomic features}

208 To understand the functional status of the various TEs in the genome, DNA-histone

209 modification location data were collected using chromatin immunoprecipitation

210 followed by sequencing (ChIP-seq) against H3K9me3 and H3K27me3, which allows

211 for the identification of DNA interacting with these modified histones.

212 Characteristics of TE sequence, such as GC percentage, composite RIP index (CRI),

213 and TE age, estimated as the Jukes-Cantor distance to the consensus sequence of the

214 specific TE family, were calculated (see methods). To further classify genomic

215 regions as eu- or heterochromatic, we performed an assay for transposase

216 accessible chromatin and sequencing (ATAC-seq) ${ }^{58}$. This method uses a TN5

217 transposase to restrict physically accessible DNA in the nucleus and tags the DNA

218 ends with oligonucleotides for downstream sequencing. Transcriptional activity was

219 assayed using RNA-sequencing. To analyze all of these TE characteristics (variables)

220 at once, dimensional reduction with principle component analysis (PCA) was

221 employed, which facilitates data interpretation on two-dimensions to identify

222 important variables and their relationships within large datasets. The individual TEs

223 were grouped into four broad classes (Type I DNA elements and Type II LTR, LINEs,

224 and Unspecified elements) and analyzed for each measured variable. The first

225 dimension of PCA shows the largest separation of the data points and variables, and

226 largely separates the data based on euchromatin versus heterochromatin features 
227 (Fig. 2A, PC1). This is seen by the variables ATAC-seq, \%GC, RNA-sequencing,

228 H3K9me3 ChIP, CRI and DNA methylation (mCG) being furthest separated along the

229 X-axis (Fig. 2A). Open chromatin features such as higher ATAC-seq, \%GC, and

230 transcriptional activity are positive on the $\mathrm{x}$-axis, with small angles between the

231 vectors, indicating correlation among those variables. Conversely, features

232 associated with heterochromatin, such as H3K9me3 association, DNA methylation

233 and indication of RIP (CRI) are all negative on the x-axis, and the position of their

234 vectors indicates correlation among these variables, and negative correlation to the

235 euchromatin features (Fig. 2A). The second axis discriminates elements based on

236 their H3K27me3 profile and sequence characteristics such as Jukes Cantor (TE age),

237 Identity and Length (Fig. 2A). For the individual element classification, there is a

238 stronger association for the LTR and Unspecified elements with the

239 heterochromatin features (Fig. 2A, grey and red ellipse extending along negative x-

240 axis). Collectively, this multivariate description of TEs identifies those that are more

241 transcribed and open as having lower association with H3K9me3, mCG, and RIP

242 mutation. There are statistically significant differences between the TE types for

243 each of these variables (Supplemental Table S2), and the LTR elements have the

244 highest levels of H3K9me3 and mCG, along with the highest CRI values and lowest

$245 \% \mathrm{GC}$, consistent with the mechanistic link between the four variables (Fig. 2B).

246 Interestingly, a bimodal distribution occurs for \%GC and CRI within the LTR and

247 Unspecified elements, indicating that some of the LTR elements have undergone RIP

248 and are heterochromatic, while other elements have not been subject to this

249 mechanism (Fig. 2B). This delineation occurs for the Unspecified and LTR elements

250 with a \%GC sequence content less than approximately 40\%, which have positive CRI

251 values and high H3K9me3 signal (Fig. 2C). A similar distinction is seen with ATAC-

252 seq data that show a clear break around 40\% GC content, and elements below this

253 have lower ATAC-seq signal and higher H3K9me3 signal (Fig. 2D). These trends are

254 not observed for the LINE and DNA elements (Supplemental Fig. S2). These results

255 suggest that LTR and Unspecified TE elements exist in two distinct chromatin states

256 in the genome. 

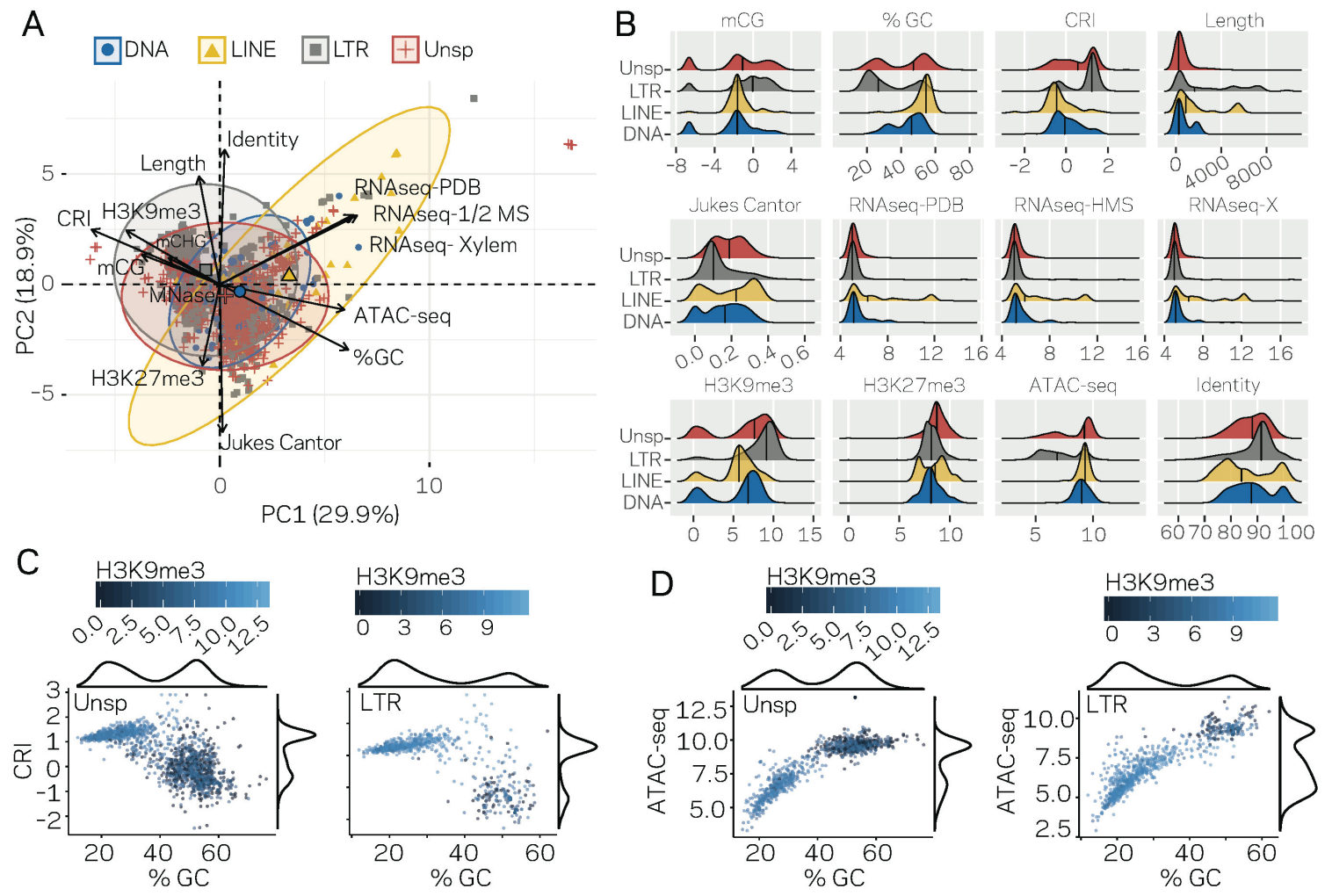

Figure 2. Individual TE families have distinct epigenetic and physical compaction profiles. (A) Principle component analysis for 14 variables measured for each individual TE. Each vector represents one variable, with the length signifying the importance of the variable in the dimension. The relationship between variables can be determined by the angle connecting two vectors. For angles $<90^{\circ}$, the two variables are correlated, while those $>90^{\circ}$ are negatively correlated. Each individual element is shown and highlighted by color and symbol as indicated by the key. Colored ellipses show the confidence interval for the four families along with a single large symbol to show the mean position for the four families. mCG, weighted CG DNA methylation; mCHG, weighted CHG DNA methylation; CRI, Composite RIP index; \%GC, percent GC sequence content; Identity, Nucleotide identity as percent identity to the consensus TE sequence of a family; Length, element length; Jukes Cantor, Jukes Cantor corrected distance as proxy of TE age; RNAseq, RNA-sequencing reads from (PDB), half strength MS (HMS) or tomato xylem sap (Xylem) grown fungus expressed as variance stabilizing transformed $\log 2$ values (see methods for details); H3K9me3, log2 (TPM+1) values of mapped reads from H3K9me3 ChIP-seq; H3K27me3, log2 (TPM+1) values of mapped reads from H3K27me3 ChIP-seq; ATAC-seq, log2 (TPM+1) values for mapped reads from Assay for transposase accessible chromatin. (B) Ridge plots showing the distribution of the individual TE families per variable. The median value is shown as a solid black line in each ridge. Variables same as in A except for mCG, log2(weighted cytosine DNA methylation + 0.01). (C) Scatter plot for \%GC versus CRI values for individual TE elements shown as points. The two plots are for TEs characterized as Unspecified (Unsp) or LTR, labeled in the upper left corner. Each point is colored according to $\log 2(\mathrm{TPM}+1)$ values from H3K9me3 ChIP-seq, scale shown above each plot. A density plot is shown for both variables on the opposite side from the labeled axis. (D) Same as in C, but the $y$-axis is now showing the log2 (TPM+1) values from ATAC-seq. 


\section{Transposable element location significantly influences the epigenetic and DNA}

\section{0 accessibility profile}

261 To further dissect the relationship between epigenetic modifications, chromatin

262 status and genomic location, pair-wise comparisons were made for all TEs in core

263 versus LS regions. All measured variables, except TE length, are significantly

264 different for TEs in the core versus LS regions (Supplemental Fig. S3). Further

265 division of the TEs indicated that the LTR and Unspecified elements showed the

266 greatest differences for core versus LS measurements (Fig. 3A), demonstrating that

267 the major driver of core versus LS differences are driven by the LTR and Unspecified

268 elements. The bimodal distribution for \%GC, CRI, H3K9me3, and ATAC-seq can be

269 accounted for in part by core versus LS separation (Fig. 3B, red versus grey).

270 Collectively, the status of the LS TE elements can be characterized as devoid of DNA
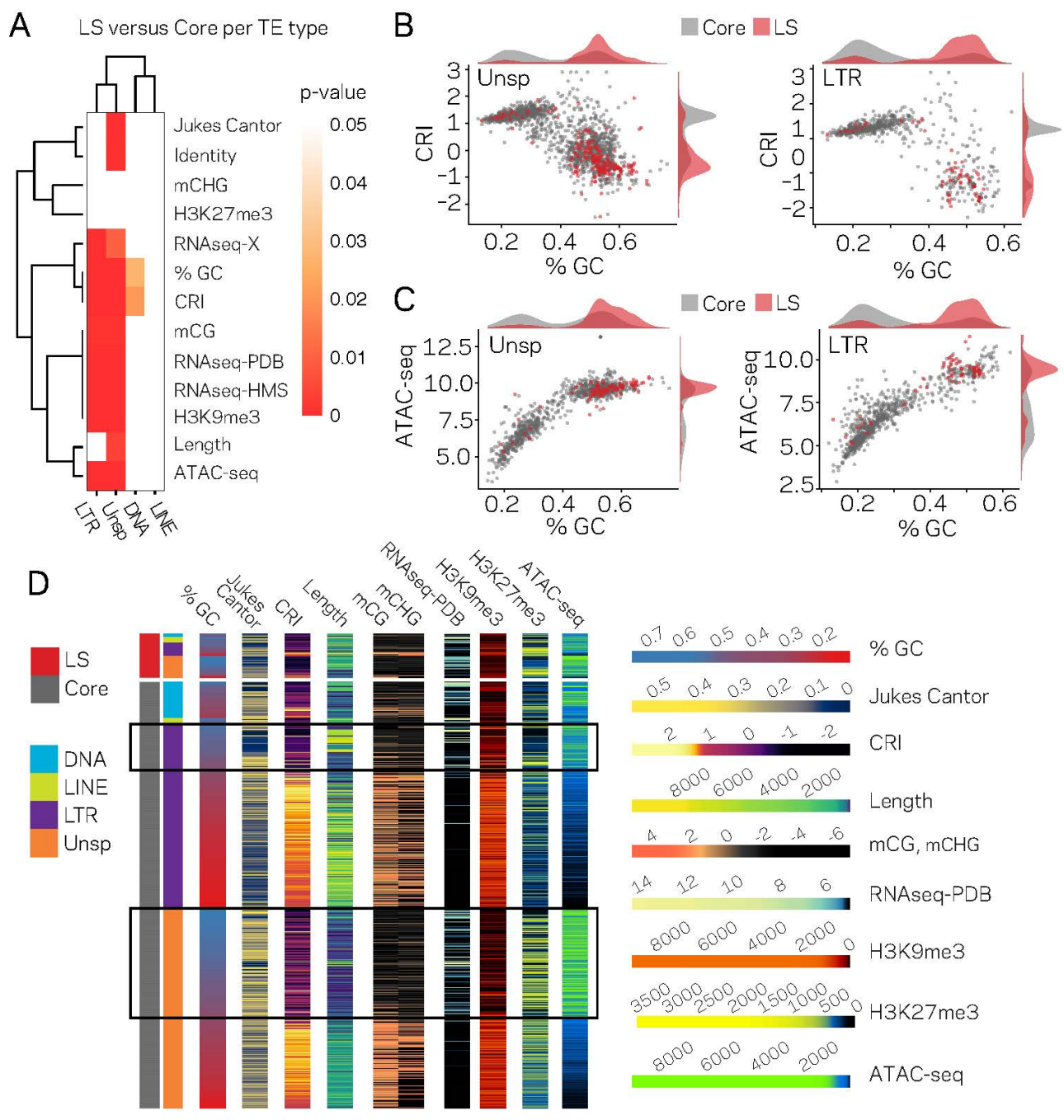
Figure 3. The LTR and Unspecified elements have significantly different chromatin profiles based on core versus LS location. (A) Heatmap comparing core versus LS values within the four TE classifications for the variable listed to the right. Plot colored based on $p$-values from Wilcoxon rank sum test. P-values $\geq 0.05$ are colored white going to red for $p$-value $\cong 0$. (B) Scatter and density plots similar to those shown in Figure 2c except the individual TE points are colored by core (grey) versus LS (red) location. The density plots are also constructed based on the two groupings (C) Similar to B, with the y-axis now showing the log2 (TPM+1) values from ATAC-seq (D) Multiple grouped heatmaps for ten variables collected for each TE. Each row represents a single element and the same ordering is used across all plots. The LS elements are grouped at the top, indicated by the red bar at the top left, and the core elements are grouped below, indicated by the grey bar at the left. Elements are further grouped by the four classifications indicated by the color code shown to the left. Within each element group, the elements are ordered by descending GC content. The scale for each heatmap is shown at the right. \% GC, percent GC sequence content; Jukes Cantor, corrected distance as proxy of TE age; CRI, Composite RIP index; Length, element length; $\mathrm{mCG}$ and $\mathrm{mCHG}, \log 2$ (weighted cytosine DNA methylation+0.01) for CG and CHG respectively; RNAseq-PDB, variance stabilizing transformed log2 RNA-sequencing reads from PDB grown fungus; $\mathrm{H} 3 \mathrm{~K} 9 \mathrm{me} 3$ and H3K27me3 and ATAC-seq, TPM values of mapped reads H3K9me3 ChIP-seq, H3K27me3 ChIP-seq, or Assay for transposase accessible chromatin respectively. Black boxes highlight LTR and Unsp elements in the core that have euchromatin profiles.

272 and H3K9 methylation, low RIP signal, generally higher than 50\% GC content, higher

273 levels of H3K27me3, more open with ATAC-seq signal, and higher transcription

274 levels (Fig. 3D). The core versus LS location is not sufficient to fully explain the 275 chromatin status, as there are many elements located in the core genome that share 276 a similar profile with the LS elements (Fig. 3D, elements highlighted in black boxes),

277 but as an ensemble, the core elements are statistically different than those found at

278 LS regions.

Significantly different chromatin status between core and LS regions extends to larger DNA segments

282 The analysis of TEs in the genome clearly shows that a subpopulation of elements

283 that occur in the previously defined LS regions have different epigenetic

284 modifications and physical openness compared to those in the core genome. LS

285 regions are significant for $V$. dahliae biology as they code many proteins which

286 support host infection. To capture a more global view of core versus LS regions, the

287 genome was analyzed using $10 \mathrm{~kb}$ non-overlapping windows, revealing the same

288 global patterns along the linear chromosome sequence; regions with high TE

289 density tend to have lower \%GC content, higher DNA and H3K9 methylation and a 
290 lack of ATAC-seq reads. The distribution of H3K27me3 appears more complicated.

291 This mark overlaps with that of DNA and H3K9 methylation, as nearly all regions

292 with these two modifications also have H3K27me3, yet we observed additional

293 regions that contain only H3K27me3 and lack DNA and H3K9 methylation (Fig. 4A).

294 The regions that contain DNA methylation and H3K9me3 are nearly identical and

295 for simplicity refer to these regions going forward as being marked by H3K9me3.

296 Interestingly, regions marked by H3K27me3 that lack H3K9me3 have more open

297 DNA than region with H3K27me3 also containing H3K9me3 (Fig. 4A, ATAC). This is

298 apparent for the LS regions that appear to have increased H3K27me3 signal, lack

299 H3K9me3 and are less open than the genomic background but not as closed as the

300 regions marked by H3K9me3 (Fig. 4B, regions marked by grey boxes). PCA was

301 again employed to combine the variables into a single analysis, with the first

302 dimension explaining nearly $60 \%$ of the variation in the data (Fig. 4C). The first

303 dimension largely captures the variables describing euchromatin versus

304 heterochromatin, such that ATAC-seq and \%GC are furthest separated on the x-axis

305 from H3K9me3, DNA methylation and TE density (Fig. 4C). Interestingly, the DNA

306 segments classified as core are mostly associated with this separation across the

307 first-dimension (Fig. 4C). The second and third dimensions of the PCA explained a

308 similar amount of variation in the data; $14.4 \%$ and $10.7 \%$, respectively. Data from

309 the RNA-seq experiment contributed nearly all the information to the second

310 dimension (Supplemental Fig. S4), while the H3K27me3 ChIP-seq data contributed

311 most of the information in the third dimension (Supplemental Table S3).

312 Interestingly, when this third dimension is considered, we observe a strong

313 separation of the core from the LS regions (Fig. 4C, y-axis), suggesting that the LS

314 regions of the genome are less defined by DNA openness, and DNA or H3K9

315 methylation but more by H3K27me3 and transcriptional activity.

316 Our observations can be summarized into a genome-wide model; for the core

317 genome, regions with higher TE density have low ATAC-seq signal (closed DNA) and

318 elevated H3K9me3 signal and thus represent the heterochromatic regions (Fig. 4D,

319 cluster of large blue dots plotted at middle left). Core genomic regions that are gene-

320 rich have a higher ATAC-seq and lower H3K9me3 signal, and represent the 
321 euchromatic portion of the genome (Fig. 4D, cluster of small blue dots plotted in the

322 lower-middle section). The LS regions are a hybrid of the two that contain high TE

323 density and higher H3K27me3 signal but have higher ATAC-seq signals when

324 compared with similar TE containing regions in the core genome (Fig. 4D, cluster of

325 large yellow triangles plotted in the middle). This simple model of the genome

326 accounts for many of the phenomena described here, and links the epigenome,

327 physical genome and functional genome.

328
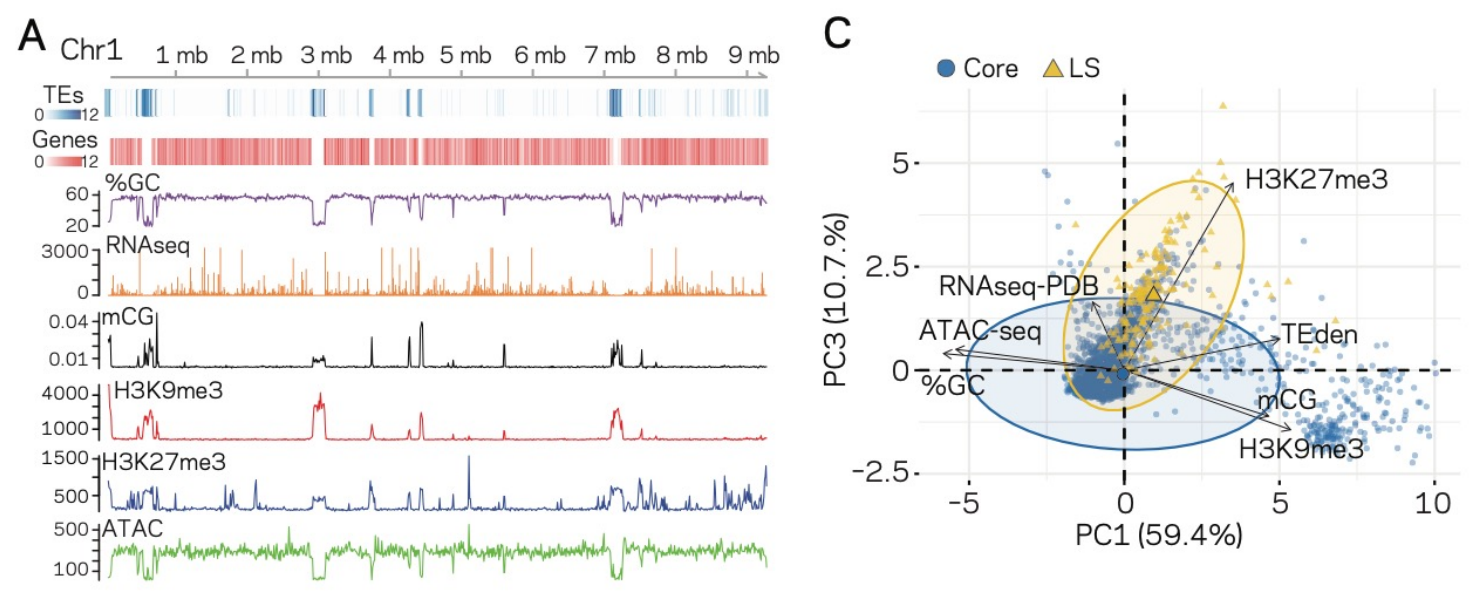

B
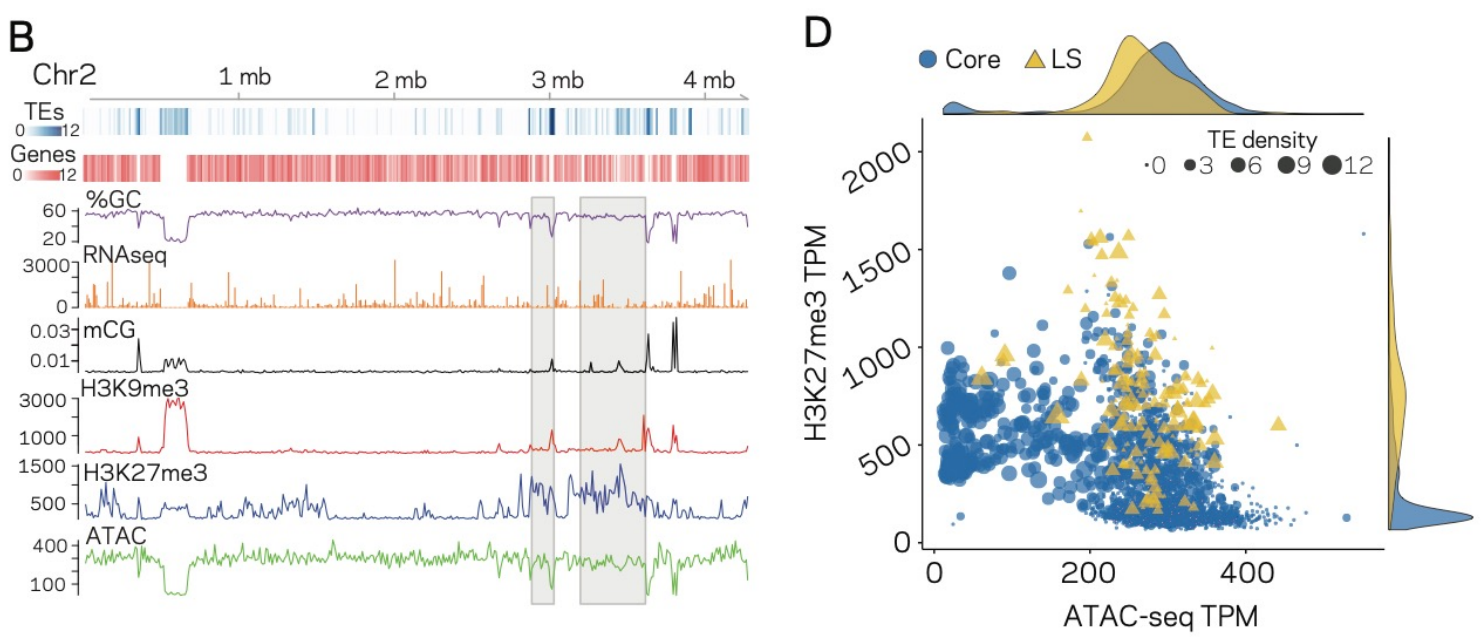

329 
Figure 4. Epigenome and physical DNA characteristics collectively define core and LS regions. (A and B) Whole chromosomes plots showing TE and gene counts over $10 \mathrm{~kb}$ genomic windows, blue and red heatmaps respectively. The \%GC content is shown in purple, RNA-seq show in orange, CG cytosine DNA methylation shown in black, H3K9me3 and H3K27me3 ChIPseq shown in red and blue respectively, and ATAC-seq shown in green. Values are those previously described. (B) Two LS regions are highlighted with a grey window. (C) Principle component analysis for seven variables at each $10 \mathrm{~kb}$ window. Dimension 1 and 3 are plotted and collective explain $\sim 70 \%$ of the variation in the data. The individual symbols are colored by genomic location with core (blue circles) and LS (yellow triangles). Colored ellipses show the confidence interval for the core and LS elements with a single large symbol to show the mean. (D) Scatter plot of the $10 \mathrm{~kb}$ windows colored for core and LS location by ATAC-seq data (TPM, $\mathrm{x}$-axis) and H3K27me3 (TPM, y-axis). The size of each symbol is proportional to the TE density shown in the upper right corner. The density plot of each variable is shown on the opposite axis.

\section{Machine learning predicts more lineage-specific genomic regions than}

333 previously considered

334 Given that a clear model emerges that links the epigenome and physical openness of

335 DNA with adaptive regions of the genome, we assessed the extent to which these

336 features can predict core or LS regions. Stimulated by our observations (Fig. 4), we

337 used ATAC-seq, RNA-seq, H3K27me3, TE density, and H3K9me3 along with the

338 binary classification of the $10 \mathrm{~kb}$ windows as core or LS for machine learning. Four

339 supervised machine learning algorithms were used to train (i.e. learn) on $80 \%$ of the

340 data (2890 regions), while the remaining 20\% (721 regions) were used for

341 prediction (i.e. test), using a 10-fold cross validation repeated three times. Assessing

342 the classifier's performance using area under the receiver operating characteristic

343 (auROC) curve suggested excellent results ranging from 0.94 to 0.95 , with a value of

3441 being perfect prediction (Fig. 5A). While auROC is the de facto standard for

345 machine learning performance 59 , it is not appropriate for assessing predictive

346 performance of binary classification problems when the two classes are heavily

347 skewed as it overestimates performance due to the high number of true negatives ${ }^{60}$.

348 This is the case for our analysis in which the test set (721 regions) contains only 33

349 of the known LS regions (4.6\%). To more accurately assess model performance,

350 precision-recall curves were employed as these do not use true negatives, and are

351 therefore less influenced by skewed binary classifications ${ }^{61}$. All four algorithms 
352 consistently outperformed a random classifier, with the boosted classification tree

353 (BCT) and stochastic gradient boosting (GMB) algorithms having the same highest

354 area under the precision-recall curve of 0.52 (Fig. 5B). However, the confusion

355 matrix indicated that the BCT model only identified 13 of the 33 LS regions (Table

356 2), resulting in poor recall (Table 3). In contrast, the other three models did identify

357 most of the known LS regions (high recall), but had lower precision caused by the

358 high rate of false positives (Table 2 and 3). The Matthews correlation coefficient

359 (MCC), an analogous measure to accuracy but more appropriate for unbalanced

360 binary classification, indicated that the GMB and random forest (RF) models

361 performed the best (Table 3).

362
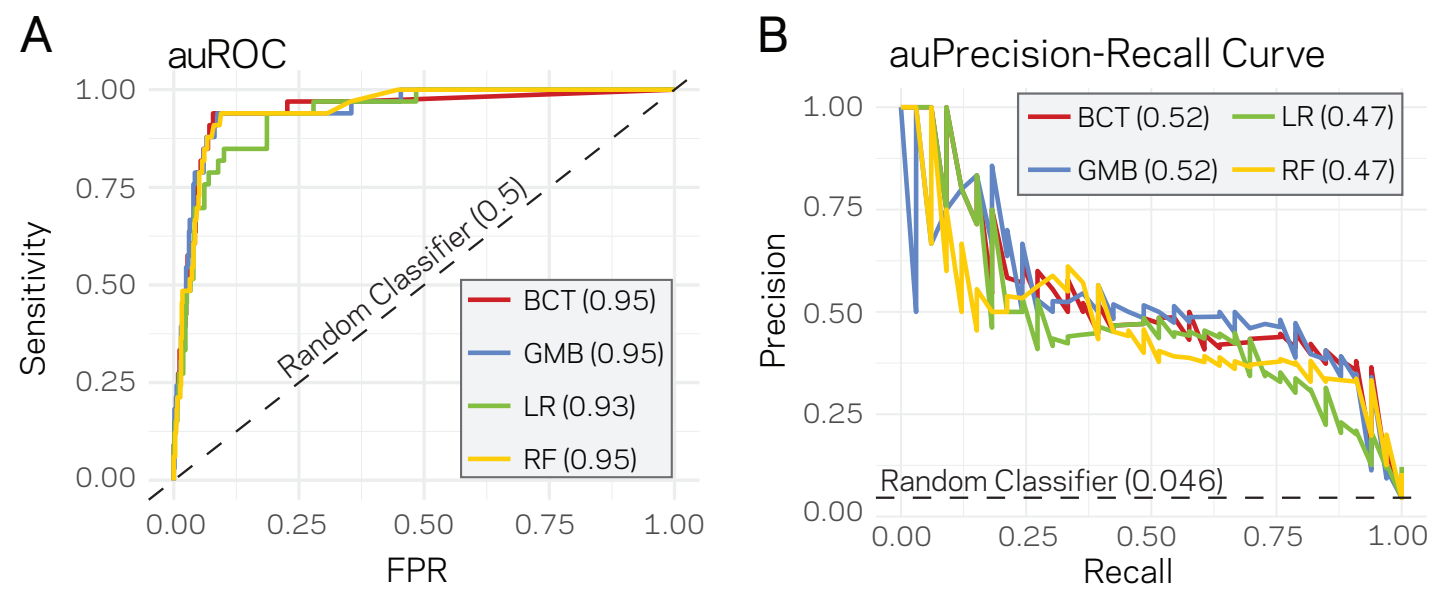

Figure 5. Supervised machine learning can predict LS regions based on epigenome and physical genome characteristics. (A) Area under the Response operator curve (auROC) plotting sensitivity and false positive rate (FPR) for four machine learning algorithms, BCT- Boosted classification tree; GMB- stochastic gradient boosting; LR- logistic regression; RF- random forest. The auROC scores are shown next the algorithm key in the grey box. The black dotted line represents the performance of a random classifier. Perfect model performance would be a curve through point $(0,1)$ in the upper left corner. (B) Area under the Precision-Recall curve for the same four models shown in A. Area under the curves are shown in the figure key in the grey box. The black dashed line shows the performance of a random classifier, calculated as the TP / (TP + FN). Perfect model performance would be a curve through point $(1,1)$ in the upper right corner. 
370 Table 2. Confusion Matrix for LS versus core prediction in V. dahliae

Known

\begin{tabular}{|c|c|c|c|}
\hline \multirow{2}{*}{\multicolumn{2}{|c|}{ Predicted }} & \multicolumn{2}{|c|}{ known } \\
\hline & & Core & $\mathrm{LS}$ \\
\hline \multirow{2}{*}{ LR } & Core & 638 & 7 \\
\hline & LS & 50 & 26 \\
\hline \multirow[t]{2}{*}{ GMB } & Core & 645 & 5 \\
\hline & LS & 43 & 28 \\
\hline \multirow{2}{*}{ BCT } & Core & 672 & 20 \\
\hline & LS & 16 & 13 \\
\hline \multirow[t]{2}{*}{$\mathrm{RF}$} & Core & 623 & 2 \\
\hline & LS & 65 & 31 \\
\hline
\end{tabular}

371 LR, Logistic Regression; GMB, Stochastic Gradient Boosting; BCT, Boosted

372 Classification Tree; RF, Random Forest; Core, regions of the genome defined as core;

373

374

LS, regions of the genome defined as Lineage Specific.

375 Table 3. Assessment values for the four tested machine learning algorithms used to 376 classify genomic regions.

\begin{tabular}{llllll}
\hline Models & Precision & Recall & MCC & F1 & F2 \\
\hline LR & 0.34 & 0.79 & 0.49 & 0.48 & 0.63 \\
GMB & 0.39 & 0.85 & 0.55 & 0.54 & 0.69 \\
BCT & 0.45 & 0.39 & 0.39 & 0.42 & 0.40 \\
RF & 0.32 & 0.94 & 0.52 & 0.48 & 0.68
\end{tabular}

LR, Logistic Regression; GMB, Stochastic Gradient Boosting; BCT, Boosted

Classification Tree; RF, Random Forest; MCC, Matthews Correlation Coefficient.

380 The results indicate that the machine learning algorithms are well-suited to identify

381 the previously known LS regions in the test data at a high rate. Additionally, the

382 algorithms identified a relatively large number of regions as LS that were previously

383 classified core. The original classification of core and LS in V. dahliae was based on

384 presence/absence variations identified from genomic information of only few

385 strains ${ }^{14,15}$. Consequently, we reasoned that regions here classified as LS by the

386 machine learning algorithms could be genuine LS regions that were originally

387 missed due to the limited diversity of the $V$. dahliae represented by the strains

388 sequenced. The two best models from the initial testing, GMB and RF, predicted a

389 total of 96 and 81 regions as LS respectively, suggesting there could be 2 to 3 times

390 more LS DNA than previously identified. To improve the genome-wide estimate and 
391 to further assess the robustness of machine learning for LS region prediction, we re-

392 ran the GMB and RF algorithms on 15 new training-test splits, independently

393 training and predicting on each set (see methods for details). This approach nearly

394 saturated the genome, providing multiple predictions per window and only 124 of

395 the 3611 regions were missed (Supplemental Fig. S5). The average MCC

396 performance estimate of the GMB and RF classifiers were 0.53 and 0.48 over the 15

397 runs, and our results indicate consistent performance across the independent

398 predictions (Fig. 6A, Supplemental Fig. S6, Supplemental Table S4 and S5). The GMB

399 classifier predicted a total of 285 of the $10 \mathrm{~kb}$ regions as LS, while the RF classifier

400 predicted 388 (Supplemental Table S6 and S7). The LS predictions for the two

401 models were in agreement for 280 regions, which is $98 \%$ of the GMB predictions

402 and $72 \%$ of those from the RF (Fig. 6B), overall showing high agreement between

403 the two classifiers. Consensus predictions were generated from the two classifiers if

404 a region was predicted as LS by both models, and a conservative joining step was

405 employed in which a single predicted core region was called LS if it was flanked by

406 LS predictions on both sides (see methods). This resulted in a total of 280 regions

407 predicted as LS by both classifiers and an additional 41 regions due to the joining. In

408 total, this new classification nearly doubles the total amount of LS regions compared

409 with the original observations ${ }^{14,15}$. The original classification of LS regions in $V$.

410 dahliae clustered in four larger regions ${ }^{14,15}$. We were interested to understand the

411 physical genomic location of the originally identified and the newly predicted LS

412 regions. The results of the individual classifiers reveal that the new regions are also

413 not randomly dispersed across the genome (Supplemental Fig. S7). The consensus

414 prediction from the two classifiers identified the large blocks of LS regions from the

415 original observations, along with new clusters of LS regions such as those on

416 chromosomes 4, 6, and 8 (Fig. 6C and 6D). Importantly, the newly defined set of LS

417 regions supports a clearer separation of the LS regions from the core regions

418 (Supplemental Fig. S8). Collectively, these analyses suggest that the machine

419 learning algorithms can be used to predict new LS regions based on epigenetic and

420 physical DNA accessibility data. The identification of potentially new LS regions 
421 missed in the original classification provides new avenues to identify proteins

422 important for host infection and adaptation. These results support that genome

423 structure is influencing genome function, demonstrates a machine learning

424 approach for predictive biology, and advances our biological understanding of

425 genome function.

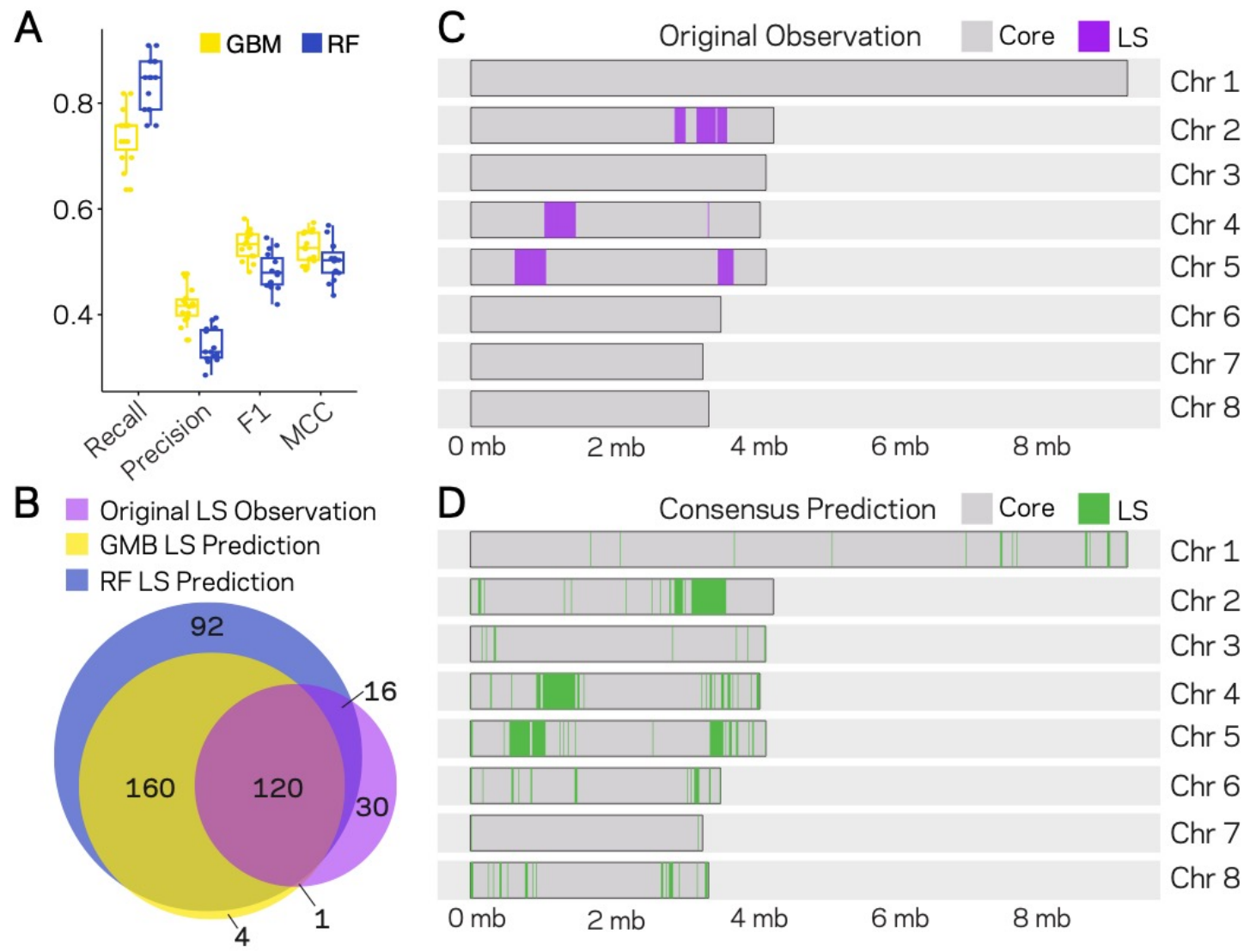

Figure 6. Machine Learning predictions for genome-wide LS content. (A) Two machine learning algorithms, Stochastic Gradient Boosting (GMB) and Random Forest (RF), were used to predict LS regions from 15 independent training-test splits. For each split, $80 \%$ of the data were used to train and the remaining $20 \%$ were used for prediction. Classifier performance was measured for each of the 15 trials, and summarized as a boxplot with each trial represented as a point. (B) Venn diagram showing the overlap between the results of the two classifiers and the original observations of LS regions (Faino et al. 2016). Each slice of the diagram shows the number of LS regions predicted, see methods for additional details. (C and D) Schematic representation of the eight chromosomes (labeled on right) of $V$. dahliae strain JR2. Each chromosome was divided into $10 \mathrm{~kb}$ windows for prediction. Regions classified as core are shown in grey and LS as their indicated color. (C) Original observations of core and LS regions, grey and purple respectively. The five main LS regions 1 can be seen on chromosome 2, 4 and 5. (D) The consensus model predictions for core and LS regions shown in grey and green respectively. The consensus predictions were those made by both the GMB and RF model (in total 280). Regions predicted as LS were joined if they were interrupted by a single window of Core prediction, adding an additional 41 LS regions for a final number of 321 LS regions in the V. dahliae JR2 genome. 


\section{DISCUSSION}

427 Significant efforts to detail genomes of filamentous pathogens, to understand

428 variation within species, and to a lesser extent to examine epigenetic modifications,

429 have increased our understanding genome function in this important group of

430 organisms ${ }^{16,54,62}$. Understanding pathogen genome evolution is of great interest to

431 help combat emerging pathogens, and to broaden our knowledge of genome biology

432 beyond model eukaryotes. Here we present a detailed analysis of the epigenome and

433 physical DNA accessibility of the vascular wilt pathogen $V$. dahliae and link these

434 analyses to previous characterizations of genomic regions contributing to host

435 colonization and adaptation ${ }^{13-16}$. A clear picture emerges in which the core genome

436 is organized into heterochromatic and euchromatic regions. The heterochromatin is

437 characterized by a high density of TEs with low GC content, high levels of DNA and

438 H3K9 methylation, low DNA accessibility and clear signatures of RIP mutations at

439 repetitive sequences. The euchromatin regions are opposite in all characteristics,

440 and this collective description is consistent with previous research in many other

441 eukaryotic genomes ${ }^{32,63,64}$. Interestingly, we provide evidence that previously

442 defined LS regions of the genome, characterized for their role in contributing to host

443 infection, exist in an intermediate chromatin state, having higher TE density than

444 the euchromatic regions, yet are devoid of DNA and H3K9 methylation.

445 Furthermore, LS regions have higher DNA accessibility than the core

446 heterochromatic regions and are more transcriptionally active, but they are less

447 accessible than the 'true' euchromatic gene-rich core regions. Notably, LS regions

448 are characterized as having a strong association with H3K27me3, similar to the

449 discovery that SM gene clusters are enriched at H3K27me3 regions in $F$.

450 graminearum ${ }^{29}$. Our results demonstrate that LS regions are by definition not

451 heterochromatic, as they are far more accessible than the true heterochromatin, and

452 yet they typically contain many heterochromatin features. We note previous

453 descriptions of contradictory heterochromatin states ${ }^{65}$, and the broad possible

454 chromatin states that may characterize a genome ${ }^{33}$. However, few previous 
455 analyses have assessed the relationship between DNA and histone modifications

456 with DNA accessibility in light of biological function of genomic adaptation.

458 Our results support the hypothesis that chromatin structure underlies genome

459 function. More specifically that chromatin modifications and DNA accessibility

460 contribute to genome evolution, not just via transcriptional control but also

461 regarding the architecture of the genome ${ }^{50}$. Along with the described associations,

462 we were able to predict LS regions using machine learning. The results of running

463 four machine learning algorithms trained on H3K9 and H3K27 methylation, RNA-

464 sequencing, TE density and DNA accessibility data, shows these variables could be

465 used to classify DNA segments as core versus LS with high recall (i.e. sensitivity).

466 The RF model showed the highest recall, correctly classifying 31 of the previously

467 observed 33 LS regions in spite of their skewed presence in the data at nearly 1:20

468 LS to core. The precision assessment of the algorithms was low because each model

469 classified regions as LS that were originally observed as core, statistically termed

470 false positives. However, the original observations represent operational

471 classification based on then available data. Consensus predictions based on the two

472 highest performing models extended the boundaries of the previous LS regions and

473 identified new potentially clustered LS regions. Thus, the use of machine learning

474 can extend our knowledge of biology and identify novel genomic regions to search

475 for as of yet uncharacterized genes with important adaptive roles. Collectively, we

476 interpret our results to indicate a strong link between the epigenome, physical DNA

477 accessibility and the occurrence of LS regions in V. dahliae. Our findings however

478 have limited inference on causation versus association, an important area for future

479 research. If there is a causative relationship between genome structure and function

480 it is interesting to consider who drives whom- do the LS regions dictate altered

481 chromatin or does altered chromatin dictate LS formation?

483 It is currently not possible to extend our machine learning predictions to additional

484 filamentous pathogen genomes, as the necessary data are not currently publicly

485 available. However, for many filamentous plant pathogens it is clear that genome 
486 variation on multiple scales, from SNPs to large structural variation, are not

487 uniformly distributed in the genome ${ }^{29}$. Recent reports from the fungal pathogen $Z$.

488 tritici addressed the role of genome stability and H3K27me3 during asexual

489 reproduction ${ }^{53,66}$. During experimental evolution, individual strains of $Z$. tritici

490 readily lose accessory chromosomes. The authors observed that a mutant lacking

491 the enzyme responsible for H3K27me3 showed less accessory chromosome loss and

492 concluded that H3K27me3 destabilizes chromosome structure ${ }^{53}$. However,

493 accessory chromosome losses were clearly biased in their individual frequency and

494 changes were not reported for core chromosomes, despite H3K27me3 being found

495 at high levels on accessory and regions of core chromosomes ${ }^{67}$. Therefore, the

496 observed genome destabilization requires additional determinants in conjunction

497 with H3K27me3 which remain to be discovered. Results presented here suggest that

498 DNA and histone methylation marks and physical DNA accessibility are important

499 additional determinants to distinguish accessory and LS regions of the genome.

500 However, we acknowledge that our model does not strictly differentiate all LS

501 region in the $V$. dahliae genome, as there are LS and core regions that have very

502 similar overall chromatin profiles, and therefore these features alone are not

503 sufficient. One factor that could explain part of this discrepancy is that LS formation

504 is likely not fully deterministic. Evolution is a stochastic process, and it seems

505 unlikely that LS formation can be described in absolute terms. Rather, it is more

506 likely to be a probabilistic process, in which specific chromatin and physical status

507 increases the likelihood for formation and maintenance of LS regions. The results

508 presented here offer an exciting new link between the epigenome, physical DNA

509 accessibility and adaptive genome evolution.

510

\section{METHODS}

\section{Fungal growth and strain construction}

513 V. dahliae strain JR2 (CBS 143773) was used for experimental analysis ${ }^{68}$. The strain

514 was maintained on potato dextrose agar (PDA) (Oxoid, Thermo Scientific, CM0139)

515 and grown at $22^{\circ} \mathrm{C}$ in the dark. For liquid grown cultures, conidiospores were 
516 collected from PDA plates after approximately two weeks and inoculated into flasks

517 containing the desired media at a concentration of $2 \times 10^{4}$ spores per mL. Media used

518 in this study include PDA, half-strength Murashige and Skoog plus vitamins (HMS)

519 (Duchefa-Biochemie, Haarlem, The Netherlands) medium supplemented with 3\%

520 sucrose and xylem sap (abbreviated, X) collected from greenhouse grown tomato

521 plants of the cultivar Moneymaker. Liquid cultures were grown for four days in the

522 dark at $22^{\circ} \mathrm{C}$ and $160 \mathrm{RPM}$. The cultures were strained through miracloth (22 $\left.\mu \mathrm{m}\right)$

523 (EMD Millipore, Darmstadt, Germany), pressed to remove liquid, flash frozen in

524 liquid nitrogen and ground to powder with a mortar and pestle. Samples were

525 stored at $-80^{\circ} \mathrm{C}$ if required prior to nucleic acid extraction.

526 The $\Delta h p 1$ strain was constructed as previously described ${ }^{69}$. Briefly, the genomic

527 DNA regions flanking the 5' and 3' HP1 coding sequence were amplified (left border,

528 For. Primer, 5'-GGTCTTAAUGACCTGAAGAATCGAGCAAGGA and

529 Rev. primer, 5'-GGCATTAAUATGAAAGCACCGGGATTTTTCT; right border,

530 For. Primer, 5'-GGACTTAAUATGCTGTTGGGAGGCAGAATAA

531 Rev. primer, 5'-GGGTTTAAUCCACGTAGATGGAGGGGTAGA). The PCR products were cloned

532 in to the pRF-HU2 vector system ${ }^{70}$ using USER enzyme following manufactured

533 protocol (New England Biolabs, MA, USA). Correctly ligated vector was transformed

534 into Agrobacterium tumefaciens strain AGL1 used for V. dahliae spore

535 transformation ${ }^{69}$. Colonies of $V$. dahliae growing on hygromycin B selection after 5

536 days were moved to individual plates containing PDA and hygromycin B. Putative

537 transformants were screened using PCR to check for deletion of the HP1 sequence

538 (For. Primer, 5'-AATCCCGCAAGGGAAAAGAGAC and Rev. primer, 5'-

539 CGTGTGCTTTGTCTTCTGACCA) and the integration of the hygromycin B sequence (For.

540 Primer, 5'- TGGAATATGCCACCAGCAGTAG and Rev. primer, 5'- GGAGTCGCATAAGGGAGAGCG) at

541 the specific locus.

542

543 Bisulfite sequencing and analysis

544 The wild-type $V$. dahliae strain and $\Delta h p 1$ were grown in liquid PDA for three days,

545 flash frozen and collected as described earlier. Extracted DNA was sent to the 
546 Beijing Genome Institute (BGI) for bisulfite conversion, library construction and

547 Illumina sequencing. Briefly, the DNA was sonicated to a fragment range of 100-300

548 bp, end-repaired and methylated sequencing adapters were ligated to 3' ends. The

549 EZ DNA Methylation-Gold kit (Zymo Research, CA, USA) was followed according to

550 manufacturer guidelines for bisulfite conversion of non-methylated DNA. Libraries

551 were paired-end 100bp sequenced on an Illumina HiSeq 2000.

552

553 Whole-genome bisulfite sequencing reads were analyzed using the BSMAP pipeline

554 (v. 2.73) and methratio script ${ }^{71}$. The results were partitioned into CG, CHG and CHH

555 cytosine sites for analysis. Only cytosine positions containing greater than 4

556 sequencing reads were included for analysis. Methylation levels were summarized

557 as weighted methylation percentage, calculated as the number of reads supporting

558 methylation over the number of cytosines sequenced or as fractional methylation,

559 calculated as the number of methylated cytosines divided by all cytosine positions

$560 \quad 72$. For fractional methylation, a cytosine was considered methylated if it was at least

$5615 \%$ methylated from all the reads covering that cytosine. As such, weighted

562 methylation captures quantitative aspects of methylation, while fractional

563 methylation is more qualitative. Weighted and fractional methylation were

564 calculated over intervals described in the text, including genes, promoters (defined

565 as the $300 \mathrm{bp}$ upstream of the translation start site), transposable elements and 10

$566 \mathrm{~kb}$ windows. For each feature, weighted and fractional methylation were calculated

567 from the sum of the mapped reads or the sum of the positions, respectively, over the

568 analyzed region. Two sample comparisons were computed using base $\mathrm{R}^{73}$ to

569 compute the non-parametric Mann-Whitney U test (equivalent to the two-sample

570 Wilcoxon rank-sum test). Principle component analyses were computed in R using

571 the packages FactoMineR (v 1.42) ${ }^{74}$ and factoextra (v 1.0.5) ${ }^{75}$.

572

573 Transposable element annotation

574 Repetitive elements were identified in the $V$. dahliae stains JR2 genome assembly ${ }^{68}$

575 as well as in three other high-quality $V$. dahliae genome assemblies ${ }^{16}$ using a 
576 combination of LTRharvest ${ }^{76}$ and LTRdigest ${ }^{77}$ followed by de novo identification of

577 RepeatModeler ${ }^{78}$. Briefly, LTR sequences were identified (recent and ancient LTR

578 insertions) and subsequently filtered, e.g. for occurrence of primer binding sites or

579 for nested insertions (see procedure outlined by Campbell and colleagues for details

580 79). Prior to the de novo prediction with RepearModeler, genome-wide occurrences

581 of the identified LTR elements are masked. Predicted LTR elements and the de novo

582 predictions from RepeatModeler were subsequently combined, and the identified

583 repeat sequences of the four V. dahliae strains were clustered using vsearch (80\%

584 sequence identity, search on both strands; v 2.4.4) ${ }^{80}$. A non-redundant V. dahliae

585 repeat library that contained consensus sequences for each cluster (i.e. repeat

586 family) was constructed by performing multiple sequence alignments using MAFFT

587 (v7.271) ${ }^{81}$ followed by the construction of a consensus sequence as described by

588 Faino et al. ${ }^{15}$. The consensus repeat library was subsequently manually curated and

589 annotated (Wicker classification ${ }^{82}$ ) using PASTEC (default databases and settings;

590 search in the reverse-complement sequence enabled) ${ }^{83}$, which is part of the REPET

591 pipeline (v2.2) ${ }^{84}$, and similarity to previously identified repetitive elements in $V$.

592 dahliae 68,85 . The occurrence and location of repeats in the genome assembly of $V$.

593 dahliae strain JR2 were determined using RepeatMasker (v 4.0.7; sensitive option).

594 The Repeatmasker output was post-processed using 'One code to find then all' 86

595 which supports the identification and combination of multiple matches (for instance

596 due to deletions or insertions) into combined, representative repeat occurrences.

597 We only further considered matches to the repeat consensus library, and thereby

598 excluded simple repeats and low-complexity regions. To estimate divergence time

599 of TEs, each individual copy of a transposable element was aligned to the consensus

600 of its family using needle, which is part of the EMBOSS package ${ }^{87}$. Sequence

601 divergence between the TEs and the TE-family consensus was corrected using the

602 Jukes-Cantor distance, with a correction term that accounts for insertions and

603 deletions ${ }^{88,89}$. The composite RIP index (CRI) was calculated as previously

604 described ${ }^{43}$. Briefly, CRI was determined by subtracting the RIP substrate from the

605 RIP product index, which are defined by dinucleotide frequencies as follows: RIP 
606 product index $=(\mathrm{TpA} / \mathrm{ApT})$ and the RIP substrate index $=(\mathrm{CpA}+\mathrm{TpG} / \mathrm{ApC}+\mathrm{GpT})$.

607 Positive CRI values indicate the analyzed sequences were subjected to the RIP

608 process. For TE analysis, elements that are less than $100 \mathrm{bp}$ were removed.

609

610 RNA-sequencing and analysis

$611 V$. dahliae strain JR2 (CBS 143773) was grown in triplicate liquid media PDB, HMS

612 and xylem sap as described. RNA extraction was carried out using TRIzol (Thermo

613 Fisher Science, Waltham, MA, USA) following manufacturer guidelines. Following

614 RNA re-suspension, contaminating DNA was removed using the TURBO DNA-free kit

615 (Ambion, Thermo Fisher Science, Waltham, MA, USA) and RNA integrity was

616 estimated by separating $2 \mu \mathrm{L}$ of each sample on a $2 \%$ agarose gel and quantified

617 using a Nanodrop (Thermo Fisher Science, Waltham, MA, USA) and stored at $-80^{\circ} \mathrm{C}$.

618 Library preparation and sequencing was carried out at BGI. Briefly, mRNA were

619 enriched based on polyadenylation purification and random hexamers were used

620 for cDNA synthesis. RNA-sequencing libraries were constructed following end-

621 repair and adapter ligation protocols and PCR amplified. Purified DNA fragments

622 were single-end 50bp sequenced on an Illumina HiSeq 2000.

624 Reads were mapped to the $V$. dahliae stain JR2 genome assembly ${ }^{68}$ using STAR (v

625 2.6.0) with settings (--sjdbGTFfeatureExon exon, --sjdbGTFtagExonParentTranscript

626 Parent, --alignIntronMax 400, --outFilterMismatchNmax 5, --outFilterIntronMotifs

627 RemoveNoncanonical) ${ }^{90}$. Mapped reads were quantified using the

628 summarizeOverlaps and variance stabilizing transformation (vst) features of

629 DESeq2 ${ }^{91}$. For TE analysis, the coordinates of the annotated TEs were used as

630 features for read counting. To perform RNAseq analysis over whole genome $10 \mathrm{~kb}$

631 regions, raw mapped reads were summed over $10 \mathrm{~kb}$ bins using bedtools (v 2.27) ${ }^{91}$

632 and converted to Transcripts Per Million (TPM) and averaged over the three reps

633 for analysis.

634

635 


\section{Chromatin immunoprecipitation and sequencing and analysis}

638 V. dahliae strain JR2 was grown in PDB and materials was collected as described.

639 Approximately $400 \mathrm{mg}$ ground material was resuspended in $4 \mathrm{ml}$ ChIP Lysis buffer

640 (50 mM HEPES-KOH pH7.5, 140 mM NaCl, 1 mM EDTA, 1\% Triton X-100, 0.1\%

641 NaDOC) and dounced 40 times in a $10 \mathrm{~cm}^{3}$ glass tube with tight fitting pestle on 800

642 power with a RZR50 homogenizer (Heidolph, Schwabach, Germany), followed by

643 five rounds of 20 seconds sonication on ice with 40 seconds rest between rounds

644 with a Soniprep 150 (MSE, London, UK). Samples were redistributed to $2 \mathrm{ml}$ tubes

645 and pelleted for 2 min at max speed in tabletop centrifuge. The supernatants were

646 combined, together with $20 \mu \mathrm{l} 1 \mathrm{M} \mathrm{CaCl} 2$ and $2.5 \mu \mathrm{l}$ MNase, and after 10 minutes of

647 incubation in a $37^{\circ} \mathrm{C}$ water bath with regular manual shaking, $80 \mu \mathrm{l} 0.5 \mathrm{M}$ EGTA was

648 added and tubes were put on ice. Samples were pre-cleared by adding $40 \mu$ l Protein

649 A Magnetic Beads (New England Biolabs, MA, United States) and rotating at $4^{\circ} \mathrm{C}$ for

$65060 \mathrm{~min}$, after which the beads were captured, $1 \mathrm{ml}$ fractions of supernatant were

651 moved to new $2 \mathrm{ml}$ tubes containing $5 \mu$ l H3K9me3 or H3K27me3 antibody

652 (ActiveMotif ; \#39765 and \#39155) respectively and incubated overnight with

653 continuous rotation at $4^{\circ} \mathrm{C}$. Subsequently, $20 \mu$ protein-A magnetic beads were

654 added and incubated for 3 hours at $4^{\circ} \mathrm{C}$, after which the beads were captured on a

655 magnetic stand and subsequently washed with $1 \mathrm{ml}$ wash buffer (50 mM Tris $\mathrm{HCl}$

656 pH 8, 1 mM EDTA, 1\% Triton X-100, 100 mM NaCL), high-salt wash buffer (50 mM

657 Tris HCl pH 8, 1 mM EDTA, 1\% Triton X-100, 350 mM NaCL), LiCl wash buffer (10

658 mM Tris HCl pH8, 1 mM EDTA, 0.5\% Triton X-100, 250 mM LiCl), TE buffer (10 mM

659 Tris $\mathrm{HCl} \mathrm{pH} \mathrm{8,} \mathrm{1mM} \mathrm{EDTA).} \mathrm{Nucleosomes} \mathrm{were} \mathrm{eluted} \mathrm{twice} \mathrm{from} \mathrm{beads} \mathrm{by} \mathrm{addition}$

660 of $100 \mu$ l pre-heated TES buffer (100 mM Tris HCl pH 8, 1\% SDS, 10 mM EDTA, 50

$661 \mathrm{mM} \mathrm{NaCl}$ ) and 10 minutes incubation at $65^{\circ} \mathrm{C} .10 \mathrm{mg} / \mathrm{ml} 2 \mu \mathrm{l}$ Proteinase $\mathrm{K}(10 \mathrm{mg}$

$662 / \mathrm{ml}$ ) was added and incubated at $65^{\circ} \mathrm{C}$ for 3 hours, followed by chloroform clean-up.

663 DNA was precipitated by addition of 2 volumes $100 \%$ ethanol, 1/10th volume $3 \mathrm{M}$

664 NaOAc pH 5.2 and 1/200th volume $120 \mathrm{mg} / \mathrm{ml}$ glycogen, and incubated overnight at

$665-20^{\circ} \mathrm{C}$. Sequencing libraries were prepared using the TruSeq ChIP Library

666 Preparation Kit (Illumina) according to instructions, but without gel purification and 
667 with use of the Velocity DNA Polymerase (BioLine, Luckenwalde, Germany) for 25

668 cycles of amplification. Single-end $125 \mathrm{bp}$ sequencing was performed on the Illumina

669 HiSeq2500 platform at KeyGene N.V. (Wageningen, the Netherlands).

670

671 Reads were mapped to the reference JR2 genome, using BWA-mem with default

672 settings ${ }^{92}$. For ChIP and ATAC-seq mapping, three regions of the genome were

673 masked due to aberrant mapping, possibly owing to sequence similarity to the

674 mitochondrial genome (chr1:1-45000, chr2:3466000-3475000, chr3:1-4200). This

675 is similar to what is described as blacklisted regions in other eukaryotic genomes ${ }^{93}$.

676 The raw mapped reads were counted either over the TE coordinates or $10 \mathrm{~kb}$

677 intervals for the two separate analyses. The raw mapped reads were converted to

678 TPM and the average of the two replicates was used for analysis.

680 Assay for Transposase-Accessible Chromatin (ATAC)-sequencing and analysis

681 The V. dahliae strain JR2 (CBS 143773) was grown in PDB liquid media as described.

682 Mycelium was collected, filtered, rinsed and flash frozen in liquid nitrogen. The

683 ATAC-seq procedure was carried out mainly as described previously ${ }^{94}$. Nuclei were

684 collected by resuspending ground mycelium in $5 \mathrm{~mL}$ of ice-cold Nuclei Isolation

685 Buffer (NIB) (100 mM NaCl, 4mM NaHSO 4 , 25mM Tris-HCl, 10mM MgSO 4 , 0.5mM

686 EDTA, 0.5\% NP-40 including protease inhibitors added at time of extraction, $2 \mathrm{mM}$

687 Phenylmethanesulfonyl fluoride (PMSF), $100 \mu \mathrm{M}$ Leupeptin, $1 \mu \mathrm{g} / \mathrm{mL}$ Pepstatin, 10

$688 \mu \mathrm{M}$ E-64). The homogenate was layered onto 10-mL of an ice-cold sucrose-Ficoll

689 gradient (bottom layer $5 \mathrm{~mL}$ of $2.5 \mathrm{M}$ sucrose in $25 \mathrm{mM}$ Tris- $\mathrm{HCl}, 5 \mathrm{~mL} 40 \%$ Ficoll 400

690 (GE Biosciences Corporation, NJ, USA)). Nuclei were separated into the lower phase

691 by centrifugation at $2000 \mathrm{~g}$ for $30 \mathrm{~min}$ at $4^{\circ} \mathrm{C}$. The upper layer was discarded and the

692 lower phase $(\sim 4 \mathrm{~mL})$ moved to another collection tube containing $5 \mathrm{~mL}$ of ice-cold

693 NIB. Nuclei were pelleted at $9000 \mathrm{~g}$ for $15 \mathrm{~min}$ at $4^{\circ} \mathrm{C}$ and re-suspended in $3 \mathrm{~mL}$ of

694 NIB. The integrity of the nuclei and their concentration in the solution were

695 estimated by DAPI staining (DAPI Dilactate $5 \mathrm{mg} / \mathrm{mL}$, used at a 1/2000 dilution for

696 visualization) and counted on a hemocytometer. A total of 200,000 nuclei were 
697 transferred to a $1.5 \mathrm{~mL}$ microfuge tube, and nuclei pelleted at $13000 \mathrm{~g}$ for $15 \mathrm{~min}$ at

$6984^{\circ} \mathrm{C}$ and resuspended in the transposition reaction $(20 \mathrm{uL}$ of $2 \mathrm{x}$ Nextera reaction

699 buffer, 0.5 uL of Nextera Tn5 Transposase, 19.5 uL of nuclease-free $\mathrm{H}_{2} 0$ ) (Illumina,

700 Nextera DNA library Preparation kit FA-121-1030) and the reaction was carried out

701 for 5 minutes at $37^{\circ} \mathrm{C}$. The reaction was halted and fragmented DNA purified using a

702 MinuElute PCR purification kit (Qiagen, MD, USA). The eluted DNA was amplified in

703 reaction buffer (10uL of transposased DNA, 10uL nuclease-free $\mathrm{H}_{2} 0,2.5 \mathrm{uL}$ forward

704 PCR primer (5'-AATGATACGGCGACCACCGAGATCTACACTCGTCGGCAGCGTCAGATGTG), 2.5uL

705 reverse PCR primer

706 (CAAGCAGAAGACGGCATACGAGATTTCTGCCTGTCTCGTGGGCTCGGAGATGT) and 25uL

707 NEBnext High-Fidelity 2x PCR Master Mix (New England Biolabs, MA, United

708 States)) using thermo-cycler conditions described in ${ }^{94}$ for a total of 9 cycles.

709 Amplified library was purified using the MinElute PCR Purification Kit (Qiagen, MD,

710 USA) and paired-end 100 bp sequenced on an Illumina HiSeq4000.

711

712 Reads were mapped to the reference JR2 genome with the described blacklisted

713 regions masked, using BWA-mem with default settings ${ }^{92}$. The mapped reads were

714 further processed to remove duplicates reads arising from library prep and

715 sequencing using Picard toolkit markDuplicates ${ }^{95}$. The mapped reads were counted

716 either over the TE coordinates or $10 \mathrm{~kb}$ intervals for the two separate analyses using

717 bedtools multicov ( $\mathrm{v} 2.27$ ) ${ }^{96}$. The reads were converted to TPM values and those

718 numbers used for analysis.

719

720 Machine Learning and assessment

721 The machine learning algorithms were implemented using the classification and

722 regression training (caret) package in $\mathrm{R}^{73,97}$. The full set of genomic data was used

723 to create a data frame comprising the genome in $10 \mathrm{~kb}$ segments as rows and the

724 individual collected variables as columns. The regions were classified as core or LS

725 based on the previous observations ${ }^{15}$. For initial model assessment and parameter

726 tuning, the data were split into $80 \%$ for training and $20 \%$ used for testing (i.e. 
727 prediction), and the proportion of core and LS regions were kept approximately

728 equal in the two splits. For parameter tuning, repeated cross-validation of 10-fold 3-

729 times was used and the best model was selected based on accuracy. Four algorithms

730 were used- logistic regression, random forest, stochastic gradient boosting, and

731 boosted classification tree. The model for all algorithms was classification = ATAC-

732 seq TPM + ChIP-H3K27me3 $3_{\text {TPM }}+$ ChIP-H3K9me3 $3_{\text {TPM }}+$ TE $_{\text {density }}+$ PDB-RNAseq

733 Logistic regression was run using method $\mathrm{glm}$, family binomial. Random forest was

734 run using method $r f$ and tuneGrid $[m t r y=(1,2,3)]$. The Stochastic Gradient Boosting

735 was implemented with method $\mathrm{gbm}$ and tuneGrid [interaction.depth $=(1,5,10)$,

736 n.trees $=(50,500,1000)$, shrinkage $=(0.001,0.01)$, n.minobsinnode $=(1,5)]$. The Bosted

737 Classification Tree was implemented unsing method $a d a$ and tuneGrid [iter=(100,

$7381000,3000)$, maxdepth $=(1,5,20), n u=(0.01)]$. Models were assessed using standard

739 metrics for data retrieval, with receiver operating and precision-recall curves

740 generated using package PRROC ${ }^{98}$.

741

742 To saturate the genome in predictions, a total of 15 new training test splits (80:20)

743 were generated, again maintaining the genome-wide proportion of core and LS

744 regions in data set. The random forest and stochastic gradient boosting classifiers

745 were used, based on their highest performance from the initial test. The settings

746 were picked based on best performance from initial testing: random forest, method

$747 \quad r f$ and tuneGrid [mtry=3]; stochastic gradient boosting, method $g b m$ and tuneGrid

748 [interaction.depth $=(5)$, n.trees $=(500)$, shrinkage $=(0.01)$, n.minobsinnode $=(5)]$. The

749 predictions for each of the 15 runs were assessed using the precision, recall and

750 MCC metrics. For each genomic region, a consensus designation was assigned based

751 on the highest occurrence of core versus LS prediction across the 15 trials. This was

752 done independently between the two models. A region was finally classified as LS or

753 core based on the majority classification across the 15 trails. For regions that had an

754 equal number of core and LS predictions, the regions were designated as core to be

755 conservative. A final high confidence LS consensus designation was determined for

756 each genomic region if it was predicted LS by both models. Regions predicted LS by

757 only one of the models were designated core. A conservative joining approach was 
758 used so that a single core region would be called LS if it were flanked by two LS

759 regions. This added 41 genomic regions $(410 \mathrm{~kb})$ to the $\mathrm{LS}$ genome.

760

\section{DATA ACCESS}

762 The sequencing data for this project are accessible from the National Center for

763 Biotechnology Information (NCBI) Sequence Read Archive (SRA) under BioProject

764 PRJN592220.

765

\section{ACKNOWLEDGEMENTS}

767 This work was supported in part by a European Molecular Biology Organization

768 postdoctoral fellowship (EMBO, ALTF 969-2013) and Human Frontier Science

769 Program Postdoctoral Fellowship (HFSP, LT000627/2014-L) to DEC. A portion of

770 the work was also carried out in the Cook lab under USDA-NIFA-PBI grant (2018-

771 67013-28492). Work in the laboratories of M.F.S and B.P.H.J.T. is supported by the

772 Research Council Earth and Life Sciences (ALW) of the Netherlands Organization of

773 Scientific Research (NWO).

774

\section{DISCLOSURE DECLARATION}

776 The authors declare no competing interests.

\section{SUPPLEMENTAL MATERIAL}

779 Supplemental Fig S1

780 Supplemental Fig S2

781 Supplemental Fig S3

782 Supplemental Fig S4

783 Supplemental Fig S5

784 Supplemental Fig S6

785 Supplemental Fig S7

786 Supplemental Fig S8

787

788

789

Supplemental Table S1

790 Supplemental Table S2

791 Supplemental Table S3

792 Supplemental Table S4 
836 17. de Jonge, R. et al. Tomato immune receptor Ve1 recognizes effector of

837

838

839

840

841

842

843

844

845

846

847

848

849

850

851

852

853

854

855

856

857

858

859

860

861

862

863

864

865

866

867

868

869

870

871

872

873

874

875

876

877

878

879 multiple fungal pathogens uncovered by genome and RNA sequencing. Proc. Natl. Acad. Sci. U.S.A. 109, 5110-5115 (2012).

18. Kombrink, A. et al. Verticillium dahliae LysM effectors differentially contribute to virulence on plant hosts. Mol. Plant Pathol. 18, 596-608 (2017).

19. Ma, L.-J. et al. Comparative genomics reveals mobile pathogenicity chromosomes in Fusarium. Nature 464, 367-373 (2010).

20. Raffaele, S. et al. Genome evolution following host jumps in the Irish potato famine pathogen lineage. Science 330, 1540-1543 (2010).

21. Rouxel, T. et al. Effector diversification within compartments of the Leptosphaeria maculans genome affected by Repeat-Induced Point mutations. Nature Communications 2, 202 (2011).

22. Goodwin, S. B. et al. Finished genome of the fungal wheat pathogen Mycosphaerella graminicola reveals dispensome structure, chromosome plasticity, and stealth pathogenesis. PLoS Genet. 7, e1002070 (2011).

23. Dutheil, J. Y. et al. A Tale of Genome Compartmentalization: The Evolution of Virulence Clusters in Smut Fungi. Genome Biol Evol 8, 681-704 (2016).

24. Tsushima, A. et al. Genomic Plasticity Mediated by Transposable Elements in the Plant Pathogenic Fungus Colletotrichum higginsianum. Genome Biol Evol 11, 1487-1500 (2019).

25. Peng, Z. et al. Effector gene reshuffling involves dispensable minichromosomes in the wheat blast fungus. PLoS Genet. 15, e1008272 (2019).

26. Dong, S., Raffaele, S. \& Kamoun, S. The two-speed genomes of filamentous pathogens: waltz with plants. Current Opinion in Genetics \& Development 35, 57-65 (2015).

27. Macheleidt, J. et al. Regulation and Role of Fungal Secondary Metabolites. Annual Review of Genetics 50, 371-392 (2016).

28. Soyer, J. L. et al. Epigenetic Control of Effector Gene Expression in the Plant Pathogenic Fungus Leptosphaeria maculans. PLoS Genet. 10, e1004227 (2014).

29. Connolly, L. R., Smith, K. M. \& Freitag, M. The Fusarium graminearum histone H3 K27 methyltransferase KMT6 regulates development and expression of secondary metabolite gene clusters. PLoS Genet. 9, e1003916 (2013).

30. Studt, L. et al. Knock-down of the methyltransferase Kmt6 relieves H3K27me3 and results in induction of cryptic and otherwise silent secondary metabolite gene clusters in Fusarium fujikuroi. Environ. Microbiol. 18, 4037-4054 (2016).

31. Chujo, T. \& Scott, B. Histone H3K9 and H3K27 methylation regulates fungal alkaloid biosynthesis in a fungal endophyte-plant symbiosis. Molecular Microbiology 92, 413-434 (2014).

32. Sexton, T. et al. Three-dimensional folding and functional organization principles of the Drosophila genome. Cell 148, 458-472 (2012). 
880 33. Riddle, N. C. et al. Plasticity in patterns of histone modifications and

881 chromosomal proteins in Drosophila heterochromatin. Genome Res. 21,

882

883 147-163 (2011).

884

885

886

887

34. Rea, S. et al. Regulation of chromatin structure by site-specific histone H3
methyltransferases. Nature 406, 593-599 (2000).

35. Cao, R. et al. Role of histone H3 lysine 27 methylation in polycomb-group

888 silencing. Science 298, 1039-1043 (2002).

36. Margueron, R. \& Reinberg, D. The Polycomb complex PRC2 and its mark in

889 life. Nature 469, 343-349 (2011).

890

37. Janssen, A., Colmenares, S. U. \& Karpen, G. H. Heterochromatin: Guardian of

891 the Genome. Annu. Rev. Cell Dev. Biol. 34, 265-288 (2018).

892

893

38. Tamaru, H. \& Selker, E. U. A histone H3 methyltransferase controls DNA
methylation in Neurospora crassa. Nature 414, 277-283 (2001).

894

895

39. Kouzminova, E. \& Selker, E. U. Dim-2 encodes a DNA methyltransferase

896 responsible for all known cytosine methylation in Neurospora. EMBO J. 20, 4309-4323 (2001).

897

40. Freitag, M., Hickey, P. C., Khlafallah, T. K., Read, N. D. \& Selker, E. U. HP1 Is

898

899 Essential for DNA Methylation in Neurospora. Mol. Cell 13, 427-434

900 (2004).

41. Honda, S. \& Selker, E. U. Direct interaction between DNA methyltransferase

901 DIM-2 and HP1 is required for DNA methylation in Neurospora crassa. Mol.

902 Cell. Biol. 28, 6044-6055 (2008).

903

904

905

906

907

908

909

42. Freitag, M., Williams, R. L., Kothe, G. O. \& Selker, E. U. A cytosine methyltransferase homologue is essential for repeat-induced point mutation in Neurospora crassa. Proc. Natl. Acad. Sci. U.S.A. 99, 8802-8807 (2002).

43. Lewis, Z. A. et al. Relics of repeat-induced point mutation direct heterochromatin formation in Neurospora crassa. Genome Res. 19, 427437 (2009).

910

911

912

913

914

915

916

917

918

919

44. Selker, E. U. et al. The methylated component of the Neurospora crassa genome. Nature 422, 893-897 (2003).

45. Ernst, J. et al. Mapping and analysis of chromatin state dynamics in nine human cell types. Nature 473, 43-49 (2011).

46. Bemer, M. \& Grossniklaus, U. Dynamic regulation of Polycomb group activity during plant development. Current Opinion in Plant Biology 15, 523-529 (2012).

47. Gaydos, L. J., Wang, W. \& Strome, S. Gene repression. H3K27me and PRC2 transmit a memory of repression across generations and during development. Science 345, 1515-1518 (2014).

920

48. Dattani, A. et al. Epigenetic analyses of planarian stem cells demonstrate

921

922 conservation of bivalent histone modifications in animal stem cells. Genome Res. 28, 1543-1554 (2018).

923

49. Freitag, M. Histone Methylation by SET Domain Proteins in Fungi. Annu. Rev. Microbiol. 71, 413-439 (2017). 
$924 \quad 50 . \quad$ Seidl, M. F., Cook, D. E. \& Thomma, B. P. H. J. Chromatin Biology Impacts

925

926

927

928

929

930

931

932

933

934

935

936

937

938

939

940

941

942

943

944

945

946

947

948

949

950

951

952

953

954

955

956

957

958

959

960

961

962

963

964

965

966

967

968 Adaptive Evolution of Filamentous Plant Pathogens. PLoS Pathog. 12, e1005920 (2016).

51. Galazka, J. M. et al. Neurospora chromosomes are organized by blocs of importin alpha-dependent heterochromatin that are largely independent of H3K9me3. Genome Res. 26, gr.203182.115-1080 (2016).

52. Klocko, A. D. et al. Normal chromosome conformation depends on subtelomeric facultative heterochromatin in Neurospora crassa. Proc. Natl. Acad. Sci. U.S.A. 113, 15048-15053 (2016).

53. Möller, M. et al. Destabilization of chromosome structure by histone H3 lysine 27 methylation. PLoS Genet. 15, e1008093 (2019).

54. Chen, H. et al. Phytophthora methylomes are modulated by $6 \mathrm{~mA}$ methyltransferases and associated with adaptive genome regions. Genome Biol. 19, 181-16 (2018).

55. Clark, S. J., Harrison, J., Paul, C. L. \& Frommer, M. High sensitivity mapping of methylated cytosines. Nucleic Acids Res. 22, 2990-2997 (1994).

56. Lister, R. \& Ecker, J. R. Finding the fifth base: Genome-wide sequencing of cytosine methylation. Genome Res. 19, 959-966 (2009).

57. Montanini, B. et al. Non-exhaustive DNA methylation-mediated transposon silencing in the black truffle genome, a complex fungal genome with massive repeat element content. Genome Biol. 15, 411-16 (2014).

58. Buenrostro, J. D., Giresi, P. G., Zaba, L. C., Chang, H. Y. \& Greenleaf, W. J. Transposition of native chromatin for fast and sensitive epigenomic profiling of open chromatin, DNA-binding proteins and nucleosome position. Nat. Methods 10, 1213-1218 (2013).

59. Bradley, A. P. The use of the area under the ROC curve in the evaluation of machine learning algorithms. Pattern Recognition 30, 1145-1159 (1997).

60. Davis, J. \& Goadrich, M. The relationship between precision-recall and ROC curves. in 148, 233-240 (ACM Press, 2006).

61. Saito, T. \& Rehmsmeier, M. The precision-recall plot is more informative than the ROC plot when evaluating binary classifiers on imbalanced datasets. PLoS ONE 10, e0118432 (2015).

62. Mondo, S. J. et al. Widespread adenine N6-methylation of active genes in fungi. Nature Genetics 49, 964-968 (2017).

63. Bannister, A. J. et al. Selective recognition of methylated lysine 9 on histone H3 by the HP1 chromo domain. Nature 410, 120-124 (2001).

64. Cam, H. P. et al. Comprehensive analysis of heterochromatin- and RNAimediated epigenetic control of the fission yeast genome. Nature Genetics 37, 809-819 (2005).

65. Huisinga, K. L., Brower-Toland, B. \& Elgin, S. C. R. The contradictory definitions of heterochromatin: transcription and silencing. Chromosoma 115, 110-122 (2006).

66. Möller, M., Habig, M., Freitag, M. \& Stukenbrock, E. H. Extraordinary Genome Instability and Widespread Chromosome Rearrangements During Vegetative Growth. Genetics 210, 517-529 (2018). 
969

970

971

972

973

974

975

976

977

978

979

980

981

982

983

984

985

986

987

988

989

990

991

992

993

994

995

996

997

998

999

1000

1001

1002

1003

1004

1005

1006

1007

1008

1009

1010

1011

1012

1013

1014

67. Schotanus, K. et al. Histone modifications rather than the novel regional centromeres of Zymoseptoria tritici distinguish core and accessory chromosomes. Epigenetics Chromatin 8, 41 (2015).

68. Faino, L. et al. Single-Molecule Real-Time Sequencing Combined with Optical Mapping Yields Completely Finished Fungal Genome. mBio 6, e00936-15 (2015).

69. Santhanam, P. in Plant Fungal Pathogens: Methods and Protocols (eds. Bolton, M. D. \& Thomma, B. P. H. J.) 509-517 (Humana Press, 2012).

70. Frandsen, R. J. N., Andersson, J. A., Kristensen, M. B. \& Giese, H. Efficient four fragment cloning for the construction of vectors for targeted gene replacement in filamentous fungi. BMC Mol Biol 9, 70-70 (2008).

71. Xi, Y. \& Li, W. BSMAP: Whole genome bisulfite sequence MAPping program. BMC Bioinformatics 10, 232-9 (2009).

72. Schultz, M. D., Schmitz, R. J. \& Ecker, J. R. 'Leveling' the playing field for analyses of single-base resolution DNA methylomes. Trends in Genetics 28, 583-585 (2012).

73. Team, R. C. R: A Language and Environment for Statistical Computing. (2019). Available at: https://www.R-project.org/

74. Le, S., Josse, J. \& Husson, F. FactoMineR: An RPackage for Multivariate Analysis. J. Stat. Soft. 25, 1-18 (2008).

75. Kassambara, A. \& Mundt, F. factoextra: Extract and Visualize the Results of Multivariate Data Analyses. (2017). Available at: https://CRAN.R-

project.org/package=factoextra. (Accessed: 13 November 2019)

76. Ellinghaus, D., Kurtz, S. \& Willhoeft, U. LTRharvest, an efficient and flexible software for de novo detection of LTR retrotransposons. BMC

Bioinformatics 9, 18-14 (2008).

77. Steinbiss, S., Willhoeft, U., Gremme, G. \& Kurtz, S. Fine-grained annotation and classification of de novo predicted LTR retrotransposons. Nucleic Acids Res. 37, 7002-7013 (2009).

78. Smit, A. \& Hubley, R. RepeatModeler Open-1.0. (2015). Available at: http://www.repeatmasker.org. (Accessed: 13 November 2019)

79. Campbell, M. S. et al. MAKER-P: A Tool kit for the rapid creation, management, and quality control of plant genome annotations. Plant Physiol. 164, 513-524 (2014).

80. Rognes, T., Flouri, T., Nichols, B., Quince, C. \& Mahé, F. VSEARCH: A versatile open source tool for metagenomics. PeerJ, e2584 (2016).

81. Katoh, K. \& Standley, D. M. MAFFT multiple sequence alignment software version 7: Improvements in performance and usability. Mol. Biol. Evol. 30, 772-780 (2013).

82. Wicker, T. et al. A unified classification system for eukaryotic transposable elements. Nature Reviews Genetics 8, 973-982 (2007).

83. Hoede, C. et al. PASTEC: An automatic transposable element classification tool. PLoS ONE 9, e91929 (2014).

84. Flutre, T., Duprat, E., Feuillet, C. \& Quesneville, H. Considering transposable element diversification in de novo annotation approaches. PLOS ONE 6, e16526 (2011). 
1015 85. Amyotte, S. G. et al. Transposable elements in phytopathogenic Verticillium

1016

1017

1018

1019

1020

1021

1022

1023

1024

1025

1026

1027

1028

1029

1030

1031

1032

1033

1034

1035

1036

1037

1038

1039

1040

1041

1042

1043

1044

1045

1046

1047

1048

1049 spp.: insights into genome evolution and inter- and intra-specific

diversification. BMC Genomics 13, 314-20 (2012).

86. Bailly-Bechet, M., Haudry, A. \& Lerat, E. 'One code to find them all': a perl tool to conveniently parse RepeatMasker output files. Mobile DNA 5, 13 (2014).

87. Rice, P., Longden, I. \& Bleasby, A. EMBOSS: the European Molecular Biology Open Software Suite. Trends Genet. 16, 276-277 (2000).

88. Jukes, T. H. \& Cantor, C. Evolution of protein molecules. Mammalian Protein Metabolism 3, 21-132. New York, Academic Press (1969)

89. Van De Peer, Y., Neefs, J.-M. \& De Wachter, R. Small ribosomal subunit RNA sequences, evolutionary relationships among different life forms, and mitochondrial origins. Journal of Molecular Evolution 30, 463-476 (1990).

90. Dobin, A. et al. STAR: ultrafast universal RNA-seq aligner. Bioinformatics 29, 15-21 (2013).

91. Love, M. I., Huber, W. \& Anders, S. Moderated estimation of fold change and dispersion for RNA-seq data with DESeq2. Genome Biol. 15, 550 (2014).

92. Li, H. Aligning sequence reads, clone sequences and assembly contigs with BWA-MEM. arXiv (2013).

93. Amemiya, H. M., Kundaje, A. \& Boyle, A. P. The ENCODE Blacklist: Identification of Problematic Regions of the Genome. Sci Rep 9, 9354 (2019).

94. Buenrostro, J. D., Wu, B., Chang, H. Y. \& Greenleaf, W. J. ATAC-seq: A Method for Assaying Chromatin Accessibility Genome-Wide. 21.29.1-21.29.9 (John Wiley \& Sons, Inc., 2015). doi:10.1002/0471142727.mb2129s109

95. Picard Toolkit. broadinstitute/picard (2018). Available at: http://broadinstitute.github.io/picard/. (Accessed: 22nd November 2019)

96. Quinlan, A. R. \& Hall, I. M. BEDTools: a flexible suite of utilities for comparing genomic features. Bioinformatics 26, 841-842 (2010).

97. Kuhn, M. Building predictive models in R using the caret package. J. Stat. Soft. 28, 1-26 (2008).

98. Grau, J., Grosse, I. \& Keilwagen, J. PRROC: computing and visualizing precision-recall and receiver operating characteristic curves in R. Bioinformatics 31, 2595-2597 (2015). 